\title{
GROWTH OF INTERSECTION NUMBERS FOR FREE GROUP AUTOMORPHISMS
}

\author{
JASON BEHRSTOCK, MLADEN BESTVINA, AND MATT CLAY
}

\begin{abstract}
For a fully irreducible automorphism $\phi$ of the free group $F_{k}$ we compute the asymptotics of the intersection number $n \mapsto i\left(T, T^{\prime} \phi^{n}\right)$ for trees $T, T^{\prime}$ in Outer space. We also obtain qualitative information about the geometry of the Guirardel core for the trees $T$ and $T^{\prime} \phi^{n}$ for $n$ large.
\end{abstract}

\section{INTRODUCTION}

Parallels between $\mathrm{GL}_{n}(\mathbb{Z})$, the mapping class group, $\mathcal{M C G}(\Sigma)$, and the outer automorphism group of a free group, $\operatorname{Out}\left(F_{k}\right)$, drive much of the current research of these groups and is particularly fruitful in the case of $\operatorname{Out}\left(F_{k}\right)$. The article [7] lists many similarities between these groups and uses known results in one category to generate questions in another. A significant example of this pedagogy is the question of the existence of a complex useful for studying the large scale geometry of $\operatorname{Out}\left(F_{k}\right)$ analogous to the spherical Tits building for $\mathrm{GL}_{n}(\mathbb{Z})$ or Harvey's curve complex 19] for the mapping class group.

The curve complex is a simplicial complex whose vertices correspond to homotopy classes of essential simple closed curves and whose simplices encode when curves can be realized disjointly on the surface. The curve complex has played a large role in the study of the mapping class group; one of the first major results was the computation of its homotopy type and its consequences for homological stability, dimension and duality properties of the mapping class group [16, 18. Another fundamental result is that the automorphism group of the curve complex is the (full) mapping class group (except for some small complexity cases) 21, 24, 26]. The curve complex has also played an important role in understanding the large scale geometry of the mapping class group [1, a key property there is that the curve complex is Gromov hyperbolic [27.

The situation with $\operatorname{Out}\left(F_{k}\right)$ seems to be much more complicated and an emphasis on a particular feature of the curve complex leads to a different analog. A discussion of some of these analogs and their basic properties is provided in 23. Without much doubt, a construction of such a complex and a proof of its hyperbolicity is the central question in the study of $\operatorname{Out}\left(F_{k}\right)$ today. In this introduction we will feature three candidate complexes.

Recall that an (outer) automorphism of $F_{k}$ is fully irreducible (sometimes called irreducible with irreducible powers (iwip)) if no conjugacy class of a proper free factor is periodic. These automorphisms are analogous to pseudo-Anosov homeomorphisms in mapping class groups. Further recall that Culler and Vogtmann's

Date: June 18, 2018.

Partially supported by NSF grants DMS-0812513 and DMS-0502441. 
Outer space, $\mathcal{C V}_{k}$, is the space of minimal free simplicial (metric) $F_{k}$-trees normalized such that the volume of the quotient graph is 1 [11. We consider the unprojectivized version as well, $c v_{k}$.

The complex of free factors of a free group. An $n$-simplex in this complex is a chain $F F_{0}<F F_{1}<\cdots<F F_{n}$ of nontrivial proper free factors in $F_{k}$ modulo simultaneous conjugacy. Hatcher and Vogtmann showed that this complex has the homotopy type of a bouquet of spheres, a result which is analogous to that of the spherical Tits building [20] and of the curve complex [17. By analogy with the curve complex situation where pseudo-Anosov homeomorphisms have unbounded orbits and other homeomorphisms have bounded orbits, Kapovich and Lustig have shown that fully irreducible automorphisms act with unbounded orbits and other automorphisms with bounded orbits 23. Kapovich and Lustig proved their result via a notion of intersection number using geodesic currents analogous to a construction of Bonahon's in the surface case [5].

The complex of (connected) subgraphs. A vertex is a nontrivial proper free factor of $F_{k}$ modulo conjugacy. A collection $F F_{i}$ of such free factors spans a simplex if they are compatible: there is a filtered graph $G_{0} \subset G_{1} \subset \cdots \subset G_{m}=G$ representing $F_{k}$ so that each $F F_{i}$ is represented by a connected component of some $G_{i}$. Just like the collection of very short curves in a hyperbolic surface (if nonempty) determines a simplex in the curve complex, leading to the homotopy equivalence between the thin part of Teichmüller space and the curve complex, so does the core of the union of very short edges of a marked metric graph (if nonempty) determine a simplex in the complex of subgraphs, leading to the homotopy equivalence between this complex and the thin part of Outer space. There is a natural embedding of the free factor complex into the subgraph complex (the vertex sets are equal) and this embedding is a quasi-isometry.

The splitting complex. This complex is a refinement of the complex of free factors, where one also provides a complementary free factor. More precisely, this is the simplicial complex whose vertex set is the set of free product decompositions $F_{k}=A * B$ (modulo simultaneous conjugation and switching of the factors), where $n+1$ free product decompositions span an $n$-simplex if they are pairwise compatible. Two free product decompositions $A * B$ and $A^{\prime} * B^{\prime}$ are compatible if there is a two-edge graph of groups decomposition $F_{k}=X * Y * Z$ such that collapsing one edge yields the decomposition $A * B$ and collapsing the other edge yields the decomposition $A^{\prime} * B^{\prime}$. The motivation for studying this complex comes from the observation that an essential simple closed curve on a surface determines a splitting of the fundamental group over $\mathbb{Z}$ (and not just one of the factors). Moreover, as we explain below, there is a hope that a proof of hyperbolicity of the curve complex generalizes to the splitting complex.

Scott and Swarup have shown that compatibility of $A * B$ and $A^{\prime} * B^{\prime}$ can be interpreted as the vanishing of an intersection number $i\left(A * B, A^{\prime} * B^{\prime}\right)$ [29. This number $i(-,-)$ is defined for any two splittings, either as an amalgamated free product or as an HNN-extension, of a finitely generated group. When the group is the fundamental group of a surface and the splittings arise from simple closed curves on the surface, this intersection number agrees with the geometric intersection between the two curves. 
Guirardel, incorporating work of [9] and [12, generalized Scott's intersection number to the setting of $G$-trees [13. More importantly, given two $G$-trees $T, T^{\prime}$, Guirardel constructed a core $\mathcal{C}\left(T \times T^{\prime}\right) \subset T \times T^{\prime}$. This core specifies a geometry for the pair of splittings in the sense that it is $\operatorname{CAT}(0)$ and it is equipped with two patterns respectively representing the splittings such that the geometric intersection number between the patterns in the quotient $\mathcal{C}\left(T \times T^{\prime}\right) / G$ is the intersection number $i\left(T, T^{\prime}\right)$. When the group is the fundamental group of a closed surface and the splittings arise from filling simple closed curves, the quotient $\mathcal{C}\left(T \times T^{\prime}\right) / G$ is the same surface endowed with the singular euclidean structure used by Bowditch in his proof of hyperbolicity of the curve complex 6. It seems promising that following a careful understanding of the geometry of the core for free product decompositions of $F_{k}$, Bowditch's proof of the hyperbolicity of the curve complex may also show hyperbolicity of the splitting complex. In fact, the goal of this paper is much more modest. Instead of attempting to understand the geometry of the core for a pair of free splittings we restrict ourselves to two points in Outer space, and the two points differ by a high power of a fully irreducible automorphism.

One of the main differences between the mapping class group and $\operatorname{Out}\left(F_{k}\right)$ is the inherent asymmetry present in Out $\left(F_{k}\right)$. This difference arises as a mapping class is represented by a homeomorphism of a surface, a symmetric object, whereas in general an outer automorphism of $F_{k}$ is not represented by a homeomorphism of a graph but merely a homotopy equivalence, which has no symmetry imposed. The asymmetry is most easily seen in expansion factors. A pseudo-Anosov homeomorphism of a surface has two measured foliations, a stable and unstable foliation, and an expansion factor $\lambda$ such that the homeomorphism multiplies the measure on the unstable foliation by the factor $\lambda$ and the measure on the stable foliation by the factor $\lambda^{-1}$ [31]. For the inverse of the homeomorphism, the role of the foliations change place and the expansion factor $\lambda$ remains the same. For a fully irreducible automorphism there is a homotopy equivalence of metric graphs $\sigma: \Gamma \rightarrow \Gamma$ representing the automorphism that maps vertices to vertices and linearly expands each edge of $\Gamma$ by the same number $\lambda$, known as the expansion factor, and all positive powers of $\sigma$ are locally injective on the interior of every edge (such maps are called train-track maps 4). However, unlike the surface case, there is no reason why the expansion factor for an automorphism to equal the expansion factor of its inverse. Indeed the following automorphism and it inverse provide an example where the two expansion factors are not equal:

$$
\begin{array}{rlrl}
a & \mapsto b & a & \mapsto c A \\
\phi: b & \mapsto c & \phi^{-1}: b & \mapsto a \\
c & \mapsto a b & c & \mapsto b
\end{array}
$$

The expansion factor for $\phi$ is approximately 1.32 and the expansion factor for $\phi^{-1}$ is approximately 1.46. We remark that Handel and Mosher have shown that the ratio between the logarithms of the expansion factors is bounded by a constant depending on $k$ [14.

Intersection numbers for $G$-trees, as intersection numbers for curves on a surface, are symmetric (first proved by Scott in 28, obvious for Guirardel's construction). They are also invariant under automorphisms: $i\left(T, T^{\prime}\right)=i\left(T \phi, T^{\prime} \phi\right)$ for any $G^{-}$ trees $T$ and $T^{\prime}$ and an automorphism $\phi$ of $G$ (compare to $i(\alpha, \beta)=i(\psi(\alpha), \psi(\beta))$ for any curves $\alpha, \beta$ on a surface and a mapping class $\psi$.). This imposes a symmetry 
on free group automorphisms. In particular, for any $F_{k}$-tree $T$ and $\phi \in \operatorname{Out}\left(F_{k}\right)$ one has $i\left(T, T \phi^{n}\right)=i\left(T \phi^{-n}, T\right)=i\left(T, T \phi^{-n}\right)$ for all $n$. This naturally leads one to inquire about the asymptotic behavior of the function $n \mapsto i\left(T, T^{\prime} \phi^{n}\right)$.

In the surface setting, for a pseudo-Anosov homeomorphism $\psi$ and any curves $\alpha$ and $\beta$ the function $n \mapsto i\left(\alpha, \psi^{n}(\beta)\right)$ behaves like $n \mapsto \lambda^{n}$ where $\lambda$ is the expansion factor of $\psi$ 31. This leads one to first guess that for a fully irreducible automorphism $\phi$ and any two $F_{k}$-trees $T, T^{\prime}$ the function $i\left(T, T^{\prime} \phi^{n}\right)$ also behaves like $\lambda^{n}$ where $\lambda$ is the expansion factor of $\phi$. But as stated above, this cannot possibly be true in general since the asymptotics of $i\left(T, T^{\prime} \phi^{n}\right)$ and $i\left(T, T^{\prime} \phi^{-n}\right)$ are the same but the expansion factors of $\phi$ and $\phi^{-1}$ need not be the same.

To state the correct result we remind the reader about the stable tree associated to a fully irreducible automorphism. For a fully irreducible automorphism $\phi \in$ $\operatorname{Out}\left(F_{k}\right)$ and any tree $T \in \mathcal{C} \mathcal{V}_{k}$ the sequence $T \phi^{n}$ has a well-defined limit in the compactification of Outer space called the stable tree [3]. The stable tree is a nonsimplicial (projectivized) $\mathbb{R}$-tree. It is geometric if it is dual to a measured foliation on a 2-complex [2, 25]. Geometricity of the stable tree is characterized by a train-track representative for $\phi$ 2]; this characterization appears as Theorem 5.1 For two functions $f, g: \mathbb{R} \rightarrow \mathbb{R}$ we write $f \sim g$ to mean that there are constants $K, C$ such that $\frac{1}{K} f(x)-C \leq g(x) \leq K f(x)+C$.

Theorem 1. Suppose $\phi \in \operatorname{Out}\left(F_{k}\right)$ is fully irreducible with expansion factor $\lambda$ and $T, T^{\prime} \in c v_{k}$. Let $T^{+}$be the stable tree for $\phi$ and let $\mu$ be the expansion factor of $\phi^{-1}$.

1. If $T^{+}$is geometric, then $i\left(T, T^{\prime} \phi^{n}\right) \sim \lambda^{n}$; else

2. if $T^{+}$is nongeometric, then $i\left(T, T^{\prime} \phi^{n}\right) \sim \lambda^{n}+\lambda^{n-1} \mu+\cdots+\lambda \mu^{n-1}+\mu^{n}$.

Remark 2. Case 2 in Theorem 1 can be simplified in two ways depending on whether or not $\lambda=\mu$. If $\lambda \neq \mu$ then $i\left(T, T^{\prime} \phi^{n}\right) \sim \max \{\lambda, \mu\}^{n}$. If $\lambda=\mu$ then $i\left(T, T^{\prime} \phi^{n}\right) \sim n \lambda^{n}$.

As a corollary we get a statement about the expansion factors for certain fully irreducible automorphisms. This corollary was first proved by Handel and Mosher.

Corollary $3(15)$. Suppose $\phi \in \operatorname{Out}\left(F_{k}\right)$ is fully irreducible with expansion factor $\lambda$. Let $T^{+}, T^{-}$be the stable trees for $\phi, \phi^{-1}$ respectively and let $\mu$ be the expansion factor of $\phi^{-1}$. If $T^{+}$is geometric and $T^{-}$is nongeometric then $\lambda>\mu$.

Proof. If $\phi$ is as in the hypotheses of the corollary then applying Theorem 1 we get: $\lambda^{n} \sim \lambda^{n}+\lambda^{n-1} \mu+\cdots+\lambda \mu^{n-1}+\mu^{n}$. Therefore $\lambda>\mu$.

By [13, Corollary 9.3] or [15, Corollary 3], if both $T^{+}$and $T^{-}$are geometric then $\phi$ is realized by a homeomorphism of a punctured surface. Therefore, the automorphism $\phi$ appearing in Corollary 3 is a parageometric automorphism, i.e. an automorphism with a geometric stable tree that is not realized by a homeomorphism of a punctured surface.

Remark 4. In view of this discussion, Theorem 1 can be more simply stated as saying that:

1. $i\left(T, T^{\prime} \phi^{n}\right) \sim \max \{\lambda, \mu\}^{n}$ if $\lambda \neq \mu$ or if $\phi$ is realized on a surface,

2. $i\left(T, T^{\prime} \phi^{n}\right) \sim n \lambda^{n}$ if $\lambda=\mu$ and $\phi$ is not realized on a surface.

We briefly outline the rest of the paper. In Section 1 we collect all of the necessary properties of Guirardel's core that we need. Our first step toward Theorem 1 is 
showing that the asymptotics of $i\left(T, T^{\prime} \phi^{n}\right)$ do not depend on the trees $T, T^{\prime} \in c v_{k}$ in Section 2, Following this, in Section 3 we derive an algorithm for computing Guirardel's core in terms of the map on ends associated to an automorphism and present an example. In Section 4, we further refine our algorithm to show that $i\left(T, T^{\prime} \phi^{n}\right)$ is comparable to the subtree spanned by $\left(f^{n}\right)^{-1}(p)$ where $f: T \rightarrow T$ is a lift of a special representative for $\phi$ called a train-track representative (Proposition 4.3). The appearance of the inverse of the train-track map explains the appearance of both expansion factors in the second case of Theorem 10 Further in Section 4 we give a quick proof showing that $i\left(T, T^{\prime} \phi^{n}\right)$ is asymptotically bounded below by $\max \{\lambda, \mu\}^{n}$.

The rest of the proof of Theorem 1 follows from the analysis in Section 5 of the subtree $T_{e}^{n}$ in Proposition 4.3 There are two cases depending on whether the stable tree $T^{+}$is geometric (Propositions 5.18 and 5.22). Putting together the three aforementioned propositions, we get Theorem 1 Finally we present examples of the core for both an automorphism with a geometric stable tree and an automorphism with a nongeometric stable tree highlighting the difference.

We conclude our introduction by recalling some standard definitions.

$\mathbb{R}$-trees: An $\mathbb{R}$-tree $T$ (or simply tree) is a metric space such that any two points $x, y \in T$ are the endpoints of a unique arc and this arc is isometric to an interval of $\mathbb{R}$ of length $d_{T}(x, y)$. In particular every $\mathbb{R}$-tree has a Lebesgue measure. A point $p$ is called a branch point if the number of connected components of $T \backslash\{p\}$ is greater than 2. An $\mathbb{R}$-tree is a simplicial tree if the set of branch points is discrete. In this case, we denote the originating and terminating vertices of an oriented edge $e \subset T$ by $o(e)$ and $t(e)$ respectively and the edge with reverse orientation by $\bar{e}$. A $G$-tree is a tree with an isometric action of $G$. We identify two $G$-trees if there is a $G$-equivariant isometry between them. A $G$-tree is trivial if there exists a global fixed point. We will always assume that $G$ is finitely generated and that $G$-trees are minimal (no proper invariant subtree).

We will briefly recall the definition of the boundary of a tree $T$. A ray is an isometry $R:[0, \infty) \rightarrow T$. Two rays are equivalent if their images lie in a bounded neighborhood of one another; an equivalence class of rays is called an end. The equivalence class of the ray $R$ is denoted $R_{\infty}$. A geodesic is an isometry $\rho:(-\infty, \infty) \rightarrow T$ to which are associated two ends denoted $\rho_{\infty}$ and $\rho_{-\infty}$. We will often identify a ray or geodesic with its image in $T$. The boundary of $T$, denoted $\partial T$, is the set of ends. If a basepoint $p \in T$ is fixed, the set of ends can be identified with the set of rays that originate at $p$.

For a $G$-tree $T$ it is well known that every element $g \in G$ either fixes a point in $T$ (elliptic) or else it has an axis $A_{g}$ (a set isometric to $\mathbb{R}$ ) on which it acts by translation (hyperbolic). The set of rays $R \subset A_{g}$ such that $g R \subset R$ defines a unique point $\omega_{T}(g) \in \partial T$.

Morphism in $c v_{k}$ : For trees $T, T^{\prime} \in c v_{k}$, by morphism $f: T \rightarrow T^{\prime}$ we mean an equivariant cellular map that linearly expands every edge of $T$ over a tight edge path in $T^{\prime}$. This definition of morphism differs from the usual definition for $\mathbb{R}$-trees. The notion of bounded cancellation arises frequently when dealing with automorphisms of free groups. There are many statements of bounded cancellation, the one of importance to us in this section is that any morphism $f: T \rightarrow T^{\prime}$ is a quasi-isometry. In particular, there exist positive constants $K, C$ such that $\frac{1}{K} d_{T}(x, y)-C \leq d_{T^{\prime}}(f(x), f(y)) \leq K d_{T}(x, y)+C$. The original statement of 
bounded cancellation, along with a proof can be found in [8]. A morphism $f: T \rightarrow$ $T^{\prime}$ descends to a (linear) Lipschitz homotopy equivalence $\sigma: \Gamma \rightarrow \Gamma^{\prime}$ where $\Gamma=$ $T / F_{k}$ and $\Gamma^{\prime}=T^{\prime} / F_{k}$. Also, a morphism $f: T \rightarrow T^{\prime}$ induces an equivariant homeomorphism $f_{\infty}: \partial T \rightarrow \partial T^{\prime}$ called the map on ends.

Notation: The edge path obtained by tightening an edge path $\alpha$ relative to its endpoints is denoted by $[\alpha]$ and the concatenation of two edge paths $\alpha$ and $\beta$ is denoted $\alpha \cdot \beta$.

Train-track representatives: We recall the basics of train-tracks, see 4 for proofs. For a metric graph $\Gamma$, a cellular homotopy equivalence $\sigma: \Gamma \rightarrow \Gamma$ that is linear on edges is a train-track map if there is a collection $\mathcal{L}$ of unordered pairs of distinct germs of adjacent edges such that

1. $\mathcal{L}$ is closed under the action of $\sigma$ and

2. for an edge $e \subset \Gamma$, any pair of germs crossed by $\sigma(e)$ is in $\mathcal{L}$.

The unordered pairs in $\mathcal{L}$ are called legal turns, an unordered pair of distinct germs of adjacent edges not in $\mathcal{L}$ is called an illegal turn. An edge path is legal if it only crosses legal turns. There is a metric on $\Gamma$ such that $\sigma$ linearly expands each edge of $\Gamma$ by the same factor $\lambda$, called the expansion factor. This factor is the PerronFrobenious eigenvalue of the transition matrix for $\sigma$, a positive eigenvector for this eigenvalue specifies the metric on $\Gamma$. Bounded cancellation implies that there is a bound on the amount of cancellation when tightening $\sigma(\alpha \cdot \beta)$ where $\alpha$ and $\beta$ are legal paths. We denote the optimal constant by $B C C(\sigma)$. As such, when $\alpha, \beta$ and $\gamma$ are legal paths, if length $(\beta)>\frac{2 B C C(\sigma)}{\lambda-1}$ then the length of $\left[\sigma^{n}(\alpha \cdot \beta \cdot \gamma)\right]$ goes to infinity as $n \rightarrow \infty$. The number $\frac{2 B C C(\sigma)}{\lambda-1}$ is called the critical constant for the map $\sigma$.

Nielsen paths: A Nielsen path $\gamma$ is a tight path with $[\sigma(\gamma)]=\gamma$. A tight path $\gamma$ is a periodic Nielsen path if $\left[\sigma^{n}(\gamma)\right]=\gamma$ for some $n>0$ and $\left[\sigma^{i}(\gamma)\right], i=0,1, \cdots, n-1$ is an orbit of (periodic) Nielsen paths. In this paper we always consider periodic Nielsen paths and for convenience usually omit the adjective "periodic". A Nielsen path is indivisible if it is not a concatenation of nontrivial Nielsen subpaths, and similarly for orbits of Nielsen paths. An indivisible Nielsen path (or iNp) always has a unique illegal turn and the two legal pieces have equal lengths.

Acknowledgements. The authors would like to thank the referee for suggestions which improved this work.

\section{The Guirardel CORE}

In this section we describe Guirardel's construction of a core $\mathcal{C}\left(T \times T^{\prime}\right) \subset T \times T^{\prime}$ for two $G$-trees $T$ and $T^{\prime}$. In addition, we introduce some notation and state some of the basic properties of cores needed for the following. Roughly speaking, the core is the essential part of $T \times T^{\prime}$ in terms of the diagonal action of $G$ on the product $T \times T^{\prime}$. The following definitions and remarks appear in [13].

Definition 1.1. Let $T$ be a tree and $p$ a point in $T$. A direction at $p$ is a connected component of $T-\{p\}$. If $T$ is simplicial and $e$ is an oriented edge of $T$, we will use the notation $\delta_{e}$ to denote the direction at $o(e)$ that contains $e$. Given two trees $T$ and $T^{\prime}$, a quadrant is a product $\delta \times \delta^{\prime}$ where $\delta \subset T$ and $\delta^{\prime} \subset T^{\prime}$ are directions. 
For a direction $\delta \subset T$, let $\delta_{\infty} \subset \partial T$ denote the set of ends determined by rays contained in $\delta$.

Definition 1.2. Let $T$ and $T^{\prime}$ be $G$-trees and fix a basepoint $\left(p, p^{\prime}\right) \in T \times T^{\prime}$. A quadrant $\delta \times \delta^{\prime} \subset T \times T^{\prime}$ is heavy if there exists a sequence of elements $g_{i} \in G$ such that:

1. $g_{i}\left(p, p^{\prime}\right) \in \delta \times \delta^{\prime}$; and

2. $d_{T}\left(p, g_{i} p\right) \rightarrow \infty$ and $d_{T^{\prime}}\left(p^{\prime}, g_{i} p^{\prime}\right) \rightarrow \infty$ as $i \rightarrow \infty$.

If the quadrant is not heavy, it is light.

Remark 1.3. The choice of basepoint is irrelevant. In particular, if the intersection of a quadrant $\delta \times \delta^{\prime}$ with the orbit of any point is a bounded set (or more generally has a bounded projection), then $\delta \times \delta^{\prime}$ is light.

Remark 1.4. Suppose we have an inclusion of quadrants $\delta \times \delta^{\prime} \subseteq \eta \times \eta^{\prime} \subset T \times T^{\prime}$. If $\delta \times \delta^{\prime}$ is heavy, then $g_{i}\left(p, p^{\prime}\right) \in \delta \times \delta^{\prime} \subseteq \eta \times \eta^{\prime}$ for some sequence of elements $g_{i} \in G$. As the second condition of Definition 1.2 does not depend on the quadrant, we see that the quadrant $\eta \times \eta^{\prime}$ is heavy.

We can now define Guirardel's core. It is the part of $T \times T^{\prime}$ that is not in any light quadrant.

Definition 1.5 (The Guirardel Core). Let $T$ and $T^{\prime}$ be minimal $G$-trees. Let $\mathcal{L}\left(T \times T^{\prime}\right)$ be the collection of light quadrants in the product $T \times T^{\prime}$. The core $\mathcal{C}\left(T \times T^{\prime}\right)$ is defined as:

$$
\mathcal{C}\left(T \times T^{\prime}\right)=T \times T^{\prime}-\bigcup_{\delta \times \delta^{\prime} \in \mathcal{L}\left(T \times T^{\prime}\right)} \delta \times \delta^{\prime} .
$$

Since the collection $\mathcal{L}\left(T \times T^{\prime}\right)$ of light quadrants is invariant with respect to the diagonal action of $G$ on $T \times T^{\prime}$, the group $G$ acts on the core $\mathcal{C}\left(T \times T^{\prime}\right)$. Guirardel defines the intersection number between two $G$-trees $T$ and $T^{\prime}$, denoted $i\left(T, T^{\prime}\right)$, as the volume of $\mathcal{C}\left(T \times T^{\prime}\right) / G$. The measure on $\mathcal{C}\left(T \times T^{\prime}\right)$ is induced from the product Lebesgue measure on $T \times T^{\prime}$. If $T$ and $T^{\prime}$ are simplicial then the intersection number $i\left(T, T^{\prime}\right)$ is the sum of the areas of the $2-$ cells in $\mathcal{C}\left(T \times T^{\prime}\right) / G$.

Guirardel shows that this intersection number agrees with the usual notion of intersection number for simple closed curves on a surface when $T$ and $T^{\prime}$ are the Bass-Serre trees for the splitting of the surface group associated to the curves. Also, Guirardel shows that this intersection number agrees with Scott's definition of intersection number for splittings [28] and relates the core $\mathcal{C}\left(T \times T^{\prime}\right)$ to various other constructions, see [13] for references and further motivation for this definition.

We now state several properties of the core proved by Guirardel that we need for the following.

Proposition 1.6 (Proposition 2.6 [13]). Let $T$ and $T^{\prime}$ be $G$-trees. The core $\mathcal{C}(T \times$ $\left.T^{\prime}\right)$ has convex fibers, i.e. the sets $\mathcal{C}\left(T \times T^{\prime}\right) \cap\{x\} \times T^{\prime}$ and $\mathcal{C}\left(T \times T^{\prime}\right) \cap T \times\left\{x^{\prime}\right\}$ are connected (possibly empty) for any $x \in T$ and $x^{\prime} \in T^{\prime}$.

Recall that a $G$-tree is irreducible if there exist two hyperbolic elements whose axes intersect in a compact set [10]. In particular any tree in $c v_{k}$ is irreducible.

Proposition 1.7 (Proposition $3.1[13)$ ). Let $T$ and $T^{\prime}$ be $G$-trees. If either $T$ or $T^{\prime}$ is irreducible, then $\mathcal{C}\left(T \times T^{\prime}\right) \neq \emptyset$. In particular if $T, T^{\prime} \in c v_{k}$ then $\mathcal{C}\left(T \times T^{\prime}\right) \neq \emptyset$. 
In [13], the above proposition is more general, but for our purposes, the above version is sufficient. Whenever the core is non-empty, there is a stronger condition for heavy quadrants.

Proposition 1.8 (Corollary 3.8 [13]). Let $T$ and $T^{\prime}$ be $G$-trees and suppose $\mathcal{C}(T \times$ $\left.T^{\prime}\right) \neq \emptyset$. Then a quadrant $\delta \times \delta^{\prime}$ is heavy if and only if there is an element $g \in G$ which is hyperbolic for both $T$ and $T^{\prime}$ such that $\omega_{T}(g) \in \partial \delta$ and $\omega_{T^{\prime}}(g) \in \partial \delta^{\prime}$.

It may happen that the core is not connected. In this case there is a procedure of adding "diagonal" edges to the core, resulting in the augmented core $\hat{\mathcal{C}}$. Adding edges to $\mathcal{C}$ does not effect the volume of $\mathcal{C} / G$ and hence does not effect the intersection number.

\section{THE CORE $\mathcal{C}\left(T \times T^{\prime}\right)$ FOR TREES IN $c v_{k}$}

In this section we give a method for computing the intersection number between two trees in $c v_{k}$ and show that the asymptotics of $n \mapsto i\left(T, T^{\prime} \phi^{n}\right)$ do not depend on the trees $T$ and $T^{\prime}$.

Lemma 2.1. Let $f: T \rightarrow T^{\prime}$ be a morphism between $T, T^{\prime} \in c v_{k}$. For two directions $\delta \subset T$ and $\delta^{\prime} \subset T^{\prime}$ the quadrant $\delta \times \delta^{\prime}$ is heavy if and only if $f_{\infty}\left(\delta_{\infty}\right) \cap \delta_{\infty}^{\prime} \neq \emptyset$.

Proof. Suppose that $\delta \times \delta^{\prime}$ is heavy. By Proposition 1.7 the core $\mathcal{C}\left(T \times T^{\prime}\right)$ is nonempty, and hence by Proposition [1.8 there is an element $x \in F_{k}$ with $\omega_{T}(x) \in \delta_{\infty}$ and $\omega_{T^{\prime}}(x) \in \delta_{\infty}^{\prime}$. As $f_{\infty}\left(\omega_{T}(x)\right)=\omega_{T^{\prime}}(x)$, we see that $f_{\infty}\left(\delta_{\infty}\right) \cap \delta_{\infty}^{\prime} \neq \emptyset$.

Conversely, suppose that $f_{\infty}\left(\delta_{\infty}\right) \cap \delta_{\infty}^{\prime} \neq \emptyset$. Let $R \subset \delta$ be a ray whose equivalence class is mapped by $f_{\infty}$ into $\delta_{\infty}^{\prime}$. We can assume that $f(R) \subset \delta^{\prime}$. As $T / F_{k}$ is a finite graph, there is a point $R_{0} \in R$ and elements $x_{i} \in F_{k}$ such that $x_{i} R_{0} \in R$ and $d_{T}\left(R_{0}, x_{i} R_{0}\right) \rightarrow \infty$ as $i \rightarrow \infty$. Now $x_{i} f\left(R_{0}\right)=f\left(x_{i} R_{0}\right) \in f(R) \subset \delta^{\prime}$ and by bounded cancellation $d_{T^{\prime}}\left(f\left(R_{0}\right), x_{i} f\left(R_{0}\right)\right) \rightarrow \infty$ as $i \rightarrow \infty$. Thus the point $\left(R_{0}, f\left(R_{0}\right)\right) \in T \times T^{\prime}$ and elements $x_{i} \in F_{k}$ witness $\delta \times \delta^{\prime}$ as a heavy quadrant.

Using the above lemma we can determine which rectangles of $T \times T^{\prime}$ are in the core $\mathcal{C}\left(T \times T^{\prime}\right)$. This is enough to compute the intersection number $i\left(T, T^{\prime}\right)$. The following definition is closely related to the notion of a (one-sided) cylinder in [22].

Definition 2.2. For an oriented edge $e \subset T$, the clopen subset of $\partial T$ consisting of equivalence classes of geodesic rays originating at $o(e)$ and containing $e$ is called a box, which we denote $\llbracket e \rrbracket$.

Lemma 2.3. Let $f: T \rightarrow T^{\prime}$ be a morphism between $T, T^{\prime} \in c v_{k}$. Given two edges $e \subset T$ and $e^{\prime} \subset T^{\prime}$, the rectangle $e \times e^{\prime} \subset T \times T^{\prime}$ is in the core $\mathcal{C}\left(T \times T^{\prime}\right)$ if and only if each of the subsets $f_{\infty}(\llbracket e \rrbracket) \cap \llbracket e^{\prime} \rrbracket, f_{\infty}(\llbracket \bar{e} \rrbracket) \cap \llbracket e^{\prime} \rrbracket, f_{\infty}(\llbracket e \rrbracket) \cap \llbracket \bar{e}^{\prime} \rrbracket$ and $f_{\infty}(\llbracket \bar{e} \rrbracket) \cap \llbracket \bar{e}^{\prime} \rrbracket$ is non-empty.

Proof. Let $\left(p, p^{\prime}\right)$ be an interior point in the rectangle $e \times e^{\prime}$. There are two directions at each of $p$ and $p^{\prime}$. These four directions combine to give us four quadrants $Q_{i}$, $i=1,2,3,4$. The important feature of these four quadrants is that any quadrant that contains the point $\left(p, p^{\prime}\right)$ must also contain one of the $Q_{i}$ 's. Thus by Remark 1.4. the $Q_{i}$ quadrants are heavy if and only if $\left(p, p^{\prime}\right)$ is in $\mathcal{C}\left(T \times T^{\prime}\right)$. As any of the four directions at $p$ and $p^{\prime}$ lie in a bounded neighborhood of one of $\delta_{e}, \delta_{\bar{e}}, \delta_{e^{\prime}}$ or $\delta_{\bar{e}^{\prime}}$, the $Q_{i}$ 's are heavy if and only if the quadrants $\delta_{e} \times \delta_{e^{\prime}}, \delta_{e} \times \delta_{\bar{e}^{\prime}}, \delta_{\bar{e}} \times \delta_{e^{\prime}}$ and $\delta_{\bar{e}} \times \delta_{\bar{e}^{\prime}}$ are heavy. Therefore by Lemma 2.1. each of the four quadrants above are heavy 
and hence $\left(p, p^{\prime}\right) \in \mathcal{C}\left(T \times T^{\prime}\right)$ if and only if the sets in the statement of the lemma are non-empty. As this is true for any point in the rectangle $e \times e^{\prime}$, this rectangle is in the core if and only if each of these four sets is non-empty.

Remark 2.4. In a similar manner, we can also determine exactly when a vertex $\left(v \times v^{\prime}\right)$, a vertical edge $\left(v \times e^{\prime}\right)$, a horizontal edge $\left(e \times v^{\prime}\right)$ or a "diagonal edge" (in the augmented core replacing twice light rectangles, see [13] for details) is in the core. The conditions are not as simple to state for vertices or horizontal and vertical edges as there can be several directions at a vertex. It is easy to see that $e \times e^{\prime}$ is a twice-light rectangle if and only if $f_{\infty}(\llbracket e \rrbracket)=\llbracket e^{\prime} \rrbracket$.

Remark 2.5. Another way to phrase Lemma 2.3 is given two edges $e \subset T$ and $e^{\prime} \subset$ $T^{\prime}$ the rectangle $e \times e^{\prime}$ is in the core $\mathcal{C}\left(T \times T^{\prime}\right)$ if and only if there exist two geodesics $\rho^{+}, \rho^{-}:(-\infty, \infty) \rightarrow T$ whose image contains $e$ such that $f_{\infty}\left(\rho_{\infty}^{+}\right), f_{\infty}\left(\rho_{-\infty}^{-}\right) \in \llbracket e^{\prime} \rrbracket$ and $f_{\infty}\left(\rho_{-\infty}^{+}\right), f_{\infty}\left(\rho_{\infty}^{-}\right) \in \llbracket \bar{e}^{\prime} \rrbracket$.

Definition 2.6. Let $e$ be an edge of $T$. The slice of the core $\mathcal{C}\left(T \times T^{\prime}\right)$ above $e$ is the set:

$$
\mathcal{C}_{e}=\left\{e^{\prime} \in T^{\prime} \mid e \times e^{\prime} \subset \mathcal{C}\left(T \times T^{\prime}\right)\right\}
$$

Similarly define the slice $\mathcal{C}_{e^{\prime}} \subset T$ for an edge $e^{\prime}$ of $T^{\prime}$.

By Proposition 1.6. the slice $\mathcal{C}_{e}$ is a subtree of $T^{\prime}$. Clearly, every rectangle in the core belongs to one of the sets $e \times \mathcal{C}_{e}$. For any interior point $p_{e} \in e$, the tree $\left\{p_{e}\right\} \times \mathcal{C}_{e}$ embeds in the quotient $\mathcal{C}\left(T \times T^{\prime}\right) / F_{k}$, as the stabilizer of any edge in $T$ is trivial. Therefore the intersection number $i\left(T, T^{\prime}\right)$ can be expressed as the sum:

$$
i\left(T, T^{\prime}\right)=\sum_{e \subset T / F_{k}} l_{T}(e) \operatorname{vol}\left(\mathcal{C}_{e}\right)
$$

where $l_{T}(e)$ is the length of the edge $e \subset T$ and $\operatorname{vol}\left(\mathcal{C}_{e}\right)$ is the sum of the lengths of the edges in $\mathcal{C}_{e}$. We are therefore interested in finding the slices $\mathcal{C}_{e}$ for a set of representative edges for $T / F_{k}$.

Using the above characterization of the core we can show that the asymptotics of $n \mapsto i\left(T, T^{\prime} \phi^{n}\right)$ only depend on the automorphism $\phi$ and not on the trees $T, T^{\prime} \in$ $c v_{k}$.

Lemma 2.7. For any $T, T^{\prime} \in c v_{k}$ there are constants $K \geq 1$ and $C \geq 0$ such that for any $T^{\prime \prime} \in c v_{k}$ and any edge $e \subset T^{\prime \prime}$ :

$$
\frac{1}{K} \operatorname{vol}\left(\mathcal{C}_{e}^{\prime}\right)-C \leq \operatorname{vol}\left(\mathcal{C}_{e}\right) \leq K \operatorname{vol}\left(\mathcal{C}_{e}^{\prime}\right)+C
$$

where $\mathcal{C}_{e}, \mathcal{C}_{e}^{\prime}$ are the respective slices of the cores $\mathcal{C}\left(T \times T^{\prime \prime}\right)$ and $\mathcal{C}\left(T^{\prime} \times T^{\prime \prime}\right)$ above the edge $e$.

Proof. As the volume of the slices $\mathcal{C}_{e}$ and $\mathcal{C}_{e}^{\prime}$ scale with proportionately with $T$ and $T^{\prime}$, we are free to assume that the length of any edges in $T$ or $T^{\prime}$ is 1 . Fix morphisms $f: T \rightarrow T^{\prime \prime}$ and $g: T^{\prime} \rightarrow T$. Then $h=f \circ g$ is a morphism from $T^{\prime}$ to $T^{\prime \prime}$. Fix an edge $e \subset T^{\prime \prime}$ and let $p_{e}$ be an interior point of $e$. First we will show that there are constants $K_{0} \geq 1$ and $C_{0} \geq 0$ that only depend on the morphism $g: T \rightarrow T^{\prime}$ such that $\frac{1}{K_{0}} \operatorname{vol}\left(\overline{\mathcal{C}_{e}^{\prime}}\right)-C_{0} \leq \operatorname{vol}\left(\mathcal{C}_{e}\right)$.

As $g: T^{\prime} \rightarrow T$ is a quasi-isometry, there is are constants $K_{1}$ and $K_{2}$ such that the following hold where $\rho$ and $\rho^{\prime}$ are geodesics in $T^{\prime}$ : 
1. If the Hausdorff distance between $\rho$ and $\rho^{\prime}$ is at least $K_{2}$ then the intersection of the geodesics $[g(\rho)]$ and $\left[g\left(\rho^{\prime}\right)\right]$ in $T$ is empty; and

2. If $\rho$ and $\rho^{\prime}$ intersect in $L$ segments of length $K_{1}$ that are separated by segments of length $K_{2}$ then the intersection of the geodesics $[g(\rho)]$ and $\left[g\left(\rho^{\prime}\right)\right]$ in $T$ contains at least $L$ edges.

For a finite subtree $X \subset T^{\prime}$, let $N(X)$ be equal to the maximum cardinality of collection of segments of length $K_{1}$ in $X$ that are pairwise distance at least $K_{2}$ apart. There exist constants $K_{0} \geq 1$ and $C_{0} \geq 0$ such that $\frac{1}{K_{0}} \operatorname{vol}(X)-C_{0} \leq N(X)$.

Let $\mathcal{A}$ denote a maximal collection of segments of length $K_{1}$ in $\mathcal{C}_{e}^{\prime}$ that are pairwise distance at least $K_{2}$ apart. Given a segment $\alpha \in \mathcal{A}$, as $\alpha \subseteq \mathcal{C}_{e}^{\prime}$, by Lemma 2.3 (see Remark 2.5) we can find two geodesics $\rho^{+}: \mathbb{R} \rightarrow T^{\prime}$ and $\rho^{-}: \mathbb{R} \rightarrow T^{\prime}$ that contain the segment $\alpha$ such that $h_{\infty}\left(\rho_{\infty}^{+}\right), h_{\infty}\left(\rho_{-\infty}^{-}\right) \in \llbracket e \rrbracket$ and $h_{\infty}\left(\rho_{-\infty}^{+}\right), h_{\infty}\left(\rho_{\infty}^{-}\right) \in$ $\llbracket \bar{e} \rrbracket$. Now let $e^{\prime} \subseteq T$ be an edge contained in the intersection of $\left[g\left(\rho^{+}\right)\right]$and $\left[g\left(\rho^{-}\right)\right]$. Thus $g_{\infty}\left(\rho_{\infty}^{+}\right), g_{\infty}\left(\rho_{\infty}^{-}\right) \in \llbracket e^{\prime} \rrbracket$ and $g_{\infty}\left(\rho_{-\infty}^{+}\right), g_{\infty}\left(\rho_{-\infty}^{-}\right) \in \llbracket e^{\prime} \rrbracket$. As $h_{\infty}=f_{\infty} \circ g_{\infty}$ we see that $f_{\infty}\left(\llbracket e^{\prime} \rrbracket\right) \cap \llbracket e \rrbracket \neq \emptyset$ and similarly for the three other sets in Lemma 2.3 hence $e^{\prime} \in \mathcal{C}_{e}$. Repeat for all other segments in $\mathcal{A}$. By construction, the edges in $T$ corresponding to the $N\left(\mathcal{C}_{e}^{\prime}\right)$ segments of $\mathcal{A}$ are disjoint, therefore $N\left(\mathcal{C}_{e}^{\prime}\right) \leq \operatorname{vol}\left(\mathcal{C}_{e}\right)$. Hence $\frac{1}{K_{0}} \operatorname{vol}\left(\mathcal{C}_{e}^{\prime}\right)-C_{0} \leq \operatorname{vol}\left(\mathcal{C}_{e}\right)$.

A similar argument using morphisms $f^{\prime}: T^{\prime} \rightarrow T^{\prime \prime}$ and $g^{\prime}: T \rightarrow T^{\prime}$ shows that there are constants $K_{0}^{\prime} \geq 1$ and $C_{0}^{\prime} \geq 0$ only depending on the morphism $g^{\prime}: T \rightarrow T^{\prime}$ such that $\frac{1}{K_{0}^{\prime}} \operatorname{vol}\left(\mathcal{C}_{e}\right)-C_{0}^{\prime} \leq \operatorname{vol}\left(\mathcal{C}_{e}^{\prime}\right)$. This proves the lemma.

From this lemma, we can show the independence of the asymptotics of $i\left(T, T^{\prime} \phi^{n}\right)$ in a special case.

Corollary 2.8. For any $T, T^{\prime} \in c v_{k}$, and any automorphism $\phi \in \operatorname{Out}\left(F_{k}\right)$, we have $i\left(T, T \phi^{n}\right) \sim i\left(T^{\prime}, T \phi^{n}\right)$.

Proof. This follows directly from (11) and Lemma 2.7 as the constants from the lemma only depend on $T$ and $T^{\prime}$ and not on $T^{\prime \prime}=T \phi^{n}$.

From this special case we easily derive the full independence using equivariance and symmetry.

Proposition 2.9. For any $T, T^{\prime}, T^{\prime \prime} \in c v_{k}$, and any automorphism $\phi \in \operatorname{Out}\left(F_{k}\right)$, we have $i\left(T, T \phi^{n}\right) \sim i\left(T^{\prime}, T^{\prime \prime} \phi^{n}\right)$.

Proof. Applying Corollary 2.8 along with equivariance and symmetry of intersection numbers, it follows that $i\left(T^{\prime}, T \phi^{n}\right)=i\left(T^{\prime} \phi^{-n}, T\right) \sim i\left(T^{\prime} \phi^{-n}, T^{\prime \prime}\right)=i\left(T^{\prime}, T^{\prime \prime} \phi^{n}\right)$. Combining this equivalence with the equivalence in Corollary 2.8 we get $i\left(T, T \phi^{n}\right) \sim$ $i\left(T^{\prime}, T \phi^{n}\right) \sim i\left(T^{\prime}, T^{\prime \prime} \phi^{n}\right)$ as desired.

\section{Slices of the CORE $\mathcal{C}\left(T \times T^{\prime}\right)$}

3.1. The map on ends for a free group automorphism. Let $f: T \rightarrow T^{\prime}$ be a morphism between $T, T^{\prime} \in c v_{k}$. This descends to a Lipschitz linear homotopy equivalence $\sigma: \Gamma \rightarrow \Gamma^{\prime}$ where $\Gamma=T / F_{k}$ and $\Gamma^{\prime}=T^{\prime} / F_{k}$. Fix a morphism $f^{\prime}: T^{\prime} \rightarrow$ $T$ such that the induced map $\sigma^{\prime}: \Gamma^{\prime} \rightarrow \Gamma$ is a homotopy inverse to $\sigma$. Homotoping $\sigma^{\prime}$ if necessary, we assume that the image of any small open neighborhood of any vertex of $\Gamma^{\prime}$ is not contained in an edge of $\Gamma$. Fix basepoints $*^{\prime} \in T^{\prime}$ and $* \in T$ such that $f^{\prime}\left(*^{\prime}\right)=*$. For simplicity, we denote the images of these basepoints in $\Gamma$ 
and $\Gamma^{\prime}$ by $*$ and $*^{\prime}$ respectively. We will use the map $\sigma^{\prime}: \Gamma^{\prime} \rightarrow \Gamma$ to find the map on ends $f_{\infty}: \partial T \rightarrow \partial T^{\prime}$. This is the inverse of the homeomorphism $f_{\infty}^{\prime}$.

Let $e$ be an (oriented) edge of $\Gamma$. Subdivide $e$ into $e_{+} e_{-}$and denote the subdivision point by $p_{e}$. Fix a tight edge path $\alpha_{e} \subset \Gamma$ from $*$ to $p_{e}$ whose final edge is $e_{+}$. The path $\alpha_{e}$ corresponds to choosing a representative lift for $e$ in $T$. For simplicity, we call this lift $e$ and the lift of $p_{e}$ contained in this edge $p_{e}$. This lift $e$ is oriented "away" from $*$, i.e., $o(e)$ separates $p_{e}$ from $*$. Consider the set of points $\Sigma_{e}=\left(\sigma^{\prime}\right)^{-1}\left(p_{e}\right) \subset \Gamma^{\prime}$. There is one point in this set for each edge of $\Gamma^{\prime}$ whose image under $\sigma^{\prime}$ crosses either $e$ or $\bar{e}$ counted with multiplicities.

For $q \in \Sigma_{e}$ there is a tight path $\gamma_{q} \subset \Gamma^{\prime}$ from $*^{\prime}$ to $q$ such that up to homotopy $\alpha_{e}=\left[\sigma^{\prime}\left(\gamma_{q}\right)\right]$. Further, the path $\gamma_{q}$ is unique as $\sigma^{\prime}$ is a homotopy equivalence. If before tightening the path $\sigma^{\prime}\left(\gamma_{q}\right)$, the final edge is $e_{+}$we assign $q$ a "+" sign. Else, the final edge is $\bar{e}_{-}$and we assign $q$ a "-" sign.

Let $\widetilde{\gamma}_{q}$ be the lift of $\gamma_{q}$ to $T^{\prime}$ that originates at $*^{\prime}$. Denote the terminal point of $\widetilde{\gamma}_{q}$ by $\tilde{q}$. Let $\widetilde{\Sigma}_{e} \subset T^{\prime}$ be the collection of the point $\tilde{q}$. In other words, $\widetilde{\Sigma}_{e}=\left(f^{\prime}\right)^{-1}\left(p_{e}\right)$. This set decomposes into $\widetilde{\Sigma}_{e}^{+}$("+" points) and $\widetilde{\Sigma}_{e}^{-}$("-" points), depending on the sign of the images of the points in $\Sigma_{e}$. Since $f^{\prime}$ is locally injective on the interior of edges, for any edge $e^{\prime} \subset T^{\prime}$, the set $\widetilde{\Sigma}_{e} \cap e^{\prime}$ is at most a single point. Also, since $f^{\prime}$ is cellular, $\widetilde{\Sigma}_{e}$ does not contain any vertices of $T^{\prime}$.

Remark 3.1. Our hypothesis that the image of any small open neighborhood of a vertex in $\Gamma^{\prime}$ by $\sigma^{\prime}$ is not contained in an edge of $\Gamma$ implies that every component of $T^{\prime}-\widetilde{\Sigma}_{e}$ is unbounded.

Example 3.2. Let $T \in c v_{2}$ be the tree with all edge lengths equal to 1 and such that $\Gamma=T / F_{2}$ is the 2 -rose whose petals are marked $a$ and $b$. We use capital letters to denote the edges with opposite orientation. Consider the automorphism $\phi \in \operatorname{Out}\left(F_{2}\right)$ given by $\phi(a)=a b, \phi(b)=b a b$. There is an obvious homotopy equivalence $\sigma^{\prime}: \Gamma \rightarrow \Gamma$ representing the automorphism $\phi$. We will carefully look at the above construction for the map $\sigma^{\prime}$.

Subdivide the edges of $\Gamma$ as described above, creating the points $p_{a}$ and $p_{b}$. Fix preferred paths $\alpha_{a}=a_{+}$and $\alpha_{b}=b_{+}$. Also subdivide $a=a_{1} a_{2} a_{3}$ with subdivision points $p_{a_{+}}, p_{a_{-}}$and $b_{+}=b_{1} b_{2}, b_{-}=b_{3} b_{4}$ with subdivision points $p_{b_{+}}, p_{b_{-}}$. We may assume $\sigma^{\prime}\left(a_{1}\right)=a_{+}, \sigma^{\prime}\left(a_{2}\right)=a_{-} b_{+}, \sigma^{\prime}\left(a_{3}\right)=b_{-}$and $\sigma^{\prime}\left(b_{1}\right)=b_{+}, \sigma^{\prime}\left(b_{2}\right)=$ $b_{-} a_{+}, \sigma^{\prime}\left(b_{3}\right)=a_{-} b_{+}, \sigma^{\prime}\left(b_{4}\right)=b_{-}$.

Therefore $\Sigma_{a}=\left\{p_{a_{+}}, p_{b}\right\}$ and $\Sigma_{b}=\left\{p_{a_{-}}, p_{b_{+}}, p_{b_{-}}\right\}$. Then $\left[\sigma^{\prime}\left(a_{1}\right)\right]=\left[a_{+}\right]=\alpha_{a}$ and $\left[\sigma^{\prime}\left(a B_{4} B_{3}\right)\right]=\left[a b B A_{-}\right]=a_{+}=\alpha_{a}$. Hence $p_{a_{+}}$is a "+" point and $p_{b}$ is a "-" point. Further $\left[\sigma^{\prime}\left(b A A_{3}\right)\right]=\left[b a b B A B_{-}\right]=b_{+}=\alpha_{b},\left[\sigma^{\prime}\left(b_{1}\right)\right]=\left[b_{+}\right]=\alpha_{b}$ and $\left[\sigma^{\prime}\left(b A B_{4}\right)\right]=\left[b a b B A B_{-}\right]=b_{+}=\alpha_{b}$. Therefore $p_{b_{+}}$is a " $"$ " point and $p_{a_{-}}, p_{b_{-}}$ are "-" points. Figure 1 shows the sets $\widetilde{\Sigma}_{a}$ and $\widetilde{\Sigma}_{b}$ in the tree $T$.

For $\tilde{q} \in \widetilde{\Sigma}_{e}$, let $e_{\tilde{q}}^{\prime}$ be the oriented edge in $T^{\prime}$ that contains $\tilde{q}$ and such that the initial segment of $e_{\tilde{q}}^{\prime}$ is contained in $\tilde{\gamma}_{q}$, i.e., $e_{\tilde{q}}^{\prime}$ is oriented away from $*^{\prime}$. A ray containing $e_{\tilde{q}}^{\prime}$ represents an end in the box $\llbracket e_{\tilde{q}}^{\prime} \rrbracket$. A ray originating from $*^{\prime}$ is mapped by $f_{\infty}^{\prime}$ into $\llbracket e \rrbracket$ if and only if it intersects $\widetilde{\Sigma}_{e}$ and the final point in $\widetilde{\Sigma}_{e}$ it intersects is a "+" point. Likewise, a ray originating from $*^{\prime}$ is mapped by $f_{\infty}^{\prime}$ into the complement of $\llbracket \bar{e} \rrbracket$ if and only if it does not intersects $\widetilde{\Sigma}_{e}$ or if the final point of $\widetilde{\Sigma_{e}}$ it intersects is a "-" point. Therefore as $f_{\infty}$ is the inverse of $f_{\infty}^{\prime}$ we can express 


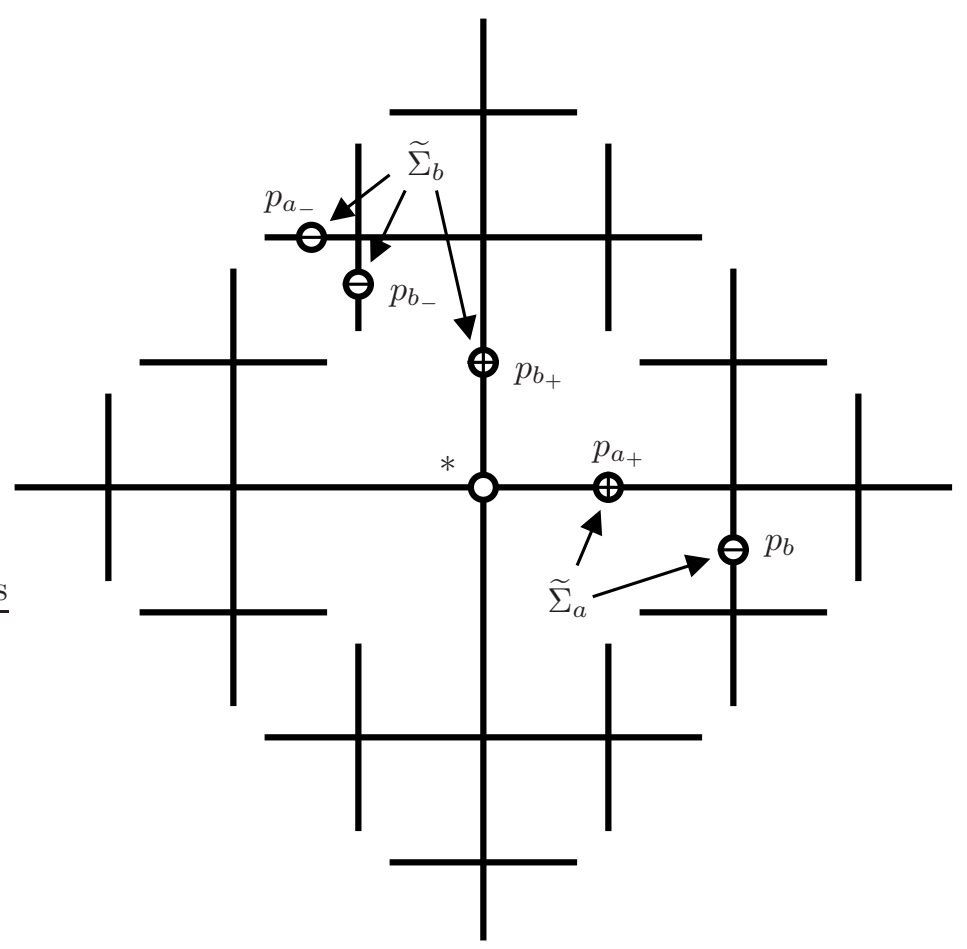

Figure 1. The sets $\widetilde{\Sigma}_{a}$ and $\widetilde{\Sigma}_{b}$ in Example 3.2. The "+" points are displayed by $\oplus$ and the "-" points are displayed by $\ominus$.

$f_{\infty}(\llbracket e \rrbracket)$ as a union and difference of the boxes $\llbracket e_{\tilde{q}}^{\prime} \rrbracket$ for $\tilde{q} \in \widetilde{\Sigma}_{e}$. See Examples 3.9 and 3.10 .

A component $X \subset T^{\prime}-\widetilde{\Sigma}_{e}$ is assigned a sign " + " if $f^{\prime}(X)$ is contained in the direction $\delta_{e} \subset T$. Else, the image $f^{\prime}(X)$ is contained in the direction $\delta_{\bar{e}} \subset T$ and we assign it a "-" sign. In the following lemma we show that along any ray, the signs of the components of $T^{\prime}-\widetilde{\Sigma}_{e}$ that the ray intersects alternate.

Lemma 3.3. With the above notation, let $R:[0, \infty) \rightarrow T^{\prime}$ be a ray that originates at a vertex of $T^{\prime}$ and suppose there are two different components $X_{0}, X_{1} \subset T^{\prime}-\widetilde{\Sigma_{e}}$ which both have the same sign and for some $0 \leq t_{0}<t_{1}$ we have $R\left(t_{0}\right) \in X_{0}$, $R\left(t_{1}\right) \in X_{1}$. Then there is a component $\widehat{X} \subset T^{\prime}-\widetilde{\Sigma}_{e}$ and $\hat{t}$ with $t_{0}<\hat{t}<t_{1}$ such that $R(\hat{t}) \in \hat{X}$ and the sign of $\widehat{X}$ is opposite to that of $X_{0}$ and $X_{1}$.

Proof. Let $x_{0}=R\left(t_{0}\right)$ and $x_{1}=R\left(t_{1}\right)$ and consider the tight segment $\gamma$ connecting $x_{0}$ to $x_{1}$, this is a subsegment of the ray $R([0, \infty))$. As $X_{0}$ and $X_{1}$ are different components of $T^{\prime}-\widetilde{\Sigma}_{e}$, for some interior point $\hat{x}$ of $\gamma$ necessarily $\hat{x} \in \widetilde{\Sigma_{e}}$. As $f^{\prime}$ maps vertices of $T^{\prime}$ to vertices of $T$, the point $\hat{x}$ is in the interior of some edge $\hat{e} \subset T^{\prime}$. As $f^{\prime}$ is injective on the interior of edges, there are points $\hat{x}_{+}, \hat{x}_{-}$in $\hat{e}$ close to $\hat{x}$ that are in components of $T^{\prime}-\widetilde{\Sigma}_{e}$ with opposite sign. One of these points gives $R(\hat{t})$.

Let $T_{e}^{\prime} \subset T^{\prime}$ be the subtree spanned by the points $\widetilde{\Sigma_{e}}$. If $e^{\prime}$ is an edge of $T^{\prime}$ that is not contained in $T_{e}^{\prime}$ and is oriented away from $T_{e}^{\prime}$, then $f_{\infty}^{\prime}\left(\llbracket e^{\prime} \rrbracket\right)$ is contained in 
either $\llbracket e \rrbracket$ or $\llbracket \bar{e} \rrbracket$. Thus either $f_{\infty}^{\prime}\left(\llbracket e^{\prime} \rrbracket\right) \cap \llbracket e \rrbracket$ or $f_{\infty}^{\prime}\left(\llbracket e^{\prime} \rrbracket\right) \cap \llbracket \bar{e} \rrbracket$ is empty and therefore by Lemma 2.3 the edge $e^{\prime}$ is not in the slice $\mathcal{C}_{e}$. Hence, the slice $\mathcal{C}_{e}$ is contained in the subtree of interior edges of $T_{e}^{\prime}$. We will show that the difference between the volume of $T_{e}^{\prime}$ and $\mathcal{C}_{e}$ is bounded, at least when then morphism $f^{\prime}: T^{\prime} \rightarrow T$ is the lift of a train-track representative.

First, let's consider a situation where an interior edge of $T_{e}^{\prime}$ is not in $\mathcal{C}_{e}$. Without loss of generality, this implies that any geodesic ray which contains this edge is eventually contained in a component of $T^{\prime}-\widetilde{\Sigma}_{e}$ that has a "+" sign. This can happen if there is a vertex of $T_{e}^{\prime}$ such that all but one of its adjacent edges contains a point in $\widetilde{\Sigma}_{e}$ which are a terminal vertices of $T_{e}^{\prime}$. In this case we would like to remove a neighborhood of this vertex and consolidate the set of points, repeating if necessary. See Figure 2

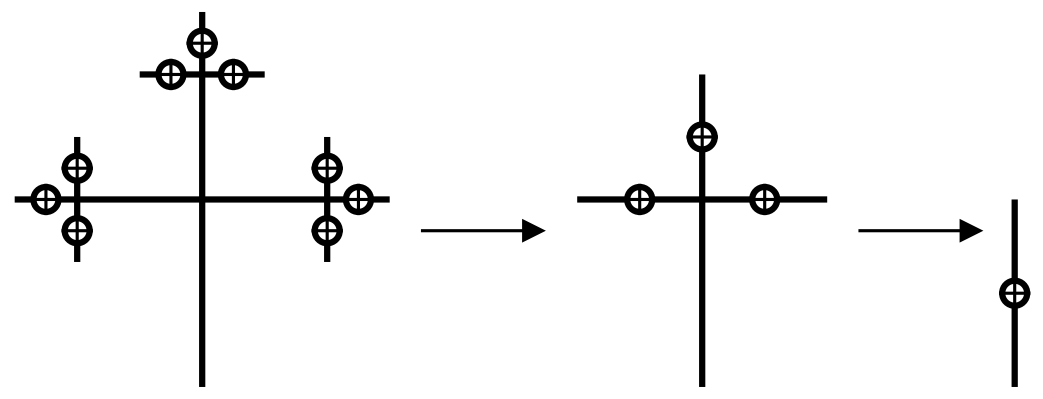

Figure 2. Consolidating vertices in $T_{e}^{\prime}$.

We now define this operation in detail and show that it terminates in the slice of the core $\mathcal{C}_{e}$. Let $V_{t}\left(T_{e}^{\prime}\right)$ denote the set of terminal vertices of $T_{e}^{\prime}$. A vertex $v \in T_{e}^{\prime}$ is full if the valence of $v$ in $T_{e}^{\prime}$ equals the valence of $v$ in $T^{\prime}$.

Definition 3.4. Let $v \in T_{e}^{\prime}$ be a full vertex with adjacent edges $\hat{e}, e_{1}, \ldots, e_{k}$ and suppose $t\left(e_{i}\right) \in V_{t}\left(T_{e}^{\prime}\right)$ for $i=1, \ldots, k$ but $t(\hat{e}) \notin V_{t}\left(T_{e}^{\prime}\right)$. Then $v$ is a removable vertex if $\left\{t\left(e_{1}\right), \ldots, t\left(e_{k}\right)\right\} \subset \widetilde{\Sigma}_{e}$.

At a removable vertex $v$, subdivide the edge $\hat{e}$ into $\hat{e}_{0} \hat{e}_{1}$ and denote the subdivision vertex by $p_{\hat{e}}$. Since the image of any small open neighborhood of $v$ is not contained in a single edge of $T$, the edge $\hat{e}$ does not contain any points in $\widetilde{\Sigma}_{e}$. Remove the $(k+1)$-pod $\hat{e}_{0}, e_{1}, \ldots, e_{k}$, leaving the vertex $p_{\hat{e}}$. Delete the points $\left\{t\left(e_{1}\right), \ldots, t\left(e_{k}\right)\right\}$ from $\widetilde{\Sigma}_{e}$ and add the point $p_{\hat{e}}$. In this new subtree $p_{\hat{e}}$ is a terminal vertex. This changes the components of $T^{\prime}-\widetilde{\Sigma}_{e}$. The components of $T^{\prime}-\left\{t\left(e_{i}\right)\right\}$ which do not contain $v$ are combined with the $(k+1)-\operatorname{pod} \hat{e}_{0}, e_{1}, \ldots, e_{k}$. The signs of all of these components were the same before the operation, and we assign this sign to the new component.

This process may create removable vertices, specifically the vertex $t(\hat{e})$. Notice that for this new collection of points $\widetilde{\Sigma}_{e}$ and components of $T^{\prime}-\widetilde{\Sigma}_{e}$, Remark 3.1 and Lemma 3.3 still hold.

Repeat this process until there are no removable vertices. As $T_{e}^{\prime}$ is a finite subtree and at each step we remove a finite subtree that does not disconnect the new space, this process will terminate with a subtree $Y_{e}^{\prime}$. In the following lemma we collect some elementary properties of the subtrees that are removed. 
Lemma 3.5. With the above notation, suppose that $A$ is a connected component of $T_{e}^{\prime}-Y_{e}^{\prime}$. Then

1. all but one of the terminal vertices of $A$ is a terminal vertex in $T_{e}^{\prime}$ and belongs to the set $\widetilde{\Sigma}_{e}$; and

2. $\widetilde{\Sigma}_{e} \cap A \subset V_{t}(A)$.

Proof. If $A$ has two terminal vertices that are not terminal vertices of $T_{e}^{\prime}$, then $Y_{e}^{\prime}$ is not connected, which is a contradiction. As all of the terminal vertices of $T_{e}^{\prime}$ belong to $\widetilde{\Sigma}_{e}, 1$. holds.

Suppose that $e^{\prime} \subset A$ contains a point of $\widetilde{\Sigma}_{e}$ that is not a terminal vertex of $T_{e}^{\prime}$. Orient $e^{\prime}$ to point away from the terminal vertex of $A$ that is not a terminal vertex of $T_{e}^{\prime}$. Then the component of $T_{e}^{\prime}-\widetilde{\Sigma}_{e}$ which contains the vertex $t\left(e^{\prime}\right)$ is bounded, which is a contradiction, hence 2 . holds.

Let $Z_{e}^{\prime}$ denote the subtree of edges in $Y_{e}^{\prime}$ not adjacent to valence one vertices, i.e., the edges of $Y_{e}^{\prime}$ that are edges in $T^{\prime}$.

Lemma 3.6. For any oriented edge $e^{\prime} \subset Z_{e}^{\prime}$ there are rays $R^{+}:[0, \infty) \rightarrow T^{\prime}$ and $R^{-}:[0, \infty) \rightarrow T^{\prime}$ containing $e^{\prime}$ with the specified orientation, such that $f_{\infty}^{\prime}\left(R_{\infty}^{+}\right) \in$ $\llbracket e \rrbracket$ and $f_{\infty}^{\prime}\left(R_{\infty}^{-}\right) \in \llbracket \bar{e} \rrbracket$.

Proof. Let $e^{\prime} \subset T_{e}^{\prime}$ and assume that for every ray $R$ containing $e^{\prime}$ we have $f_{\infty}^{\prime}\left(R_{\infty}\right) \in$ $\llbracket \bar{e} \rrbracket$. Let $A$ be the component of $T_{e}^{\prime}-o\left(e^{\prime}\right)$ that contains $e^{\prime}$. In particular, components of $T^{\prime}-\widetilde{\Sigma}_{e}$ contained in $\llbracket e^{\prime} \rrbracket$ that are adjacent to $A$ have sign "-". We will show that $A$ is removed from $T_{e}^{\prime}$ in the construction of $Z_{e}^{\prime}$ and hence $e^{\prime}$ is not in $Z_{e}^{\prime}$.

Let $X$ be the component of $T^{\prime}-\widetilde{\Sigma}_{e}$ that contains $t\left(e^{\prime}\right)$ and $X_{0}$ the component of $X-\{o(e)\}$ that contains $e^{\prime}$. We claim that $A=X_{0}$.

If there is a point in $A$ that is not in $X_{0}$, then $A$ contains a point in $\widetilde{\Sigma}_{e}$ that is not a terminal vertex of $A$. Let $x$ be such a point that is closest to a terminal vertex of $A$ and $X^{\prime}$ the component of $T^{\prime}-\widetilde{\Sigma}_{e}$ adjacent to both $x$ and a terminal vertex of $A$. As this component is unbounded, we can find a ray that contains $e^{\prime}$ and is eventually contained in $X^{\prime}$. By hypotheses, the sign of this component is "+" (as it is adjacent to a component with a "-" sign), and hence there is a ray $R$ containing $e^{\prime}$ such that $f_{\infty}^{\prime}\left(R_{\infty}\right) \in \llbracket e \rrbracket$, which contradicts our assumptions.

If $X_{0}$ is bounded then every ray containing $e^{\prime}$ intersects some point in $\widetilde{\Sigma}_{e}$. Hence, if there is a point in $X_{0}$ that is not in $A$ then $X_{0}$ is unbounded and therefore $X$ has sign "-". But then $X_{0}$ is also adjacent to a component of $T^{\prime}-\widetilde{\Sigma}_{e}$ with a "+" sign and as before there is a ray $R$ containing $e^{\prime}$ such that $f_{\infty}^{\prime}\left(R_{\infty}\right) \in \llbracket e \rrbracket$, which contradicts our assumptions.

Therefore as vertices of $X_{0}$ that are vertices of $T^{\prime}$ are full, vertices of $A$ adjacent to terminal vertices of $A$ are removable. We can repeat to see that $A$ is removed by removing removable vertices and edges adjacent to valence one vertices.

Hence, if $e^{\prime} \subset Z_{e}^{\prime}$, not every ray containing $e^{\prime}$ is mapped by $f_{\infty}^{\prime}$ to $\llbracket e^{\prime} \rrbracket$. Similarly, we see that for $e^{\prime} \subset Z_{e}^{\prime}$, not every ray that contains $e^{\prime}$ is mapped by $f_{\infty}^{\prime}$ to $\llbracket e \rrbracket$.

Lemma 3.7. For any edge $e \subset T$ the slice $\mathcal{C}_{e}$ of the core $\mathcal{C}\left(T \times T^{\prime}\right)$ is the subtree $Z_{e}^{\prime} \subset T^{\prime}$.

Proof. We have already seen that $\mathcal{C}_{e} \subseteq T_{e}^{\prime}$. By Lemma 3.5 we see that for any edge $e^{\prime} \subset T_{e}^{\prime}-Z_{e}^{\prime}$ one of the sets $f_{\infty}\left(\llbracket e^{\prime} \rrbracket\right)$ or $f_{\infty}\left(\llbracket \bar{e}^{\prime} \rrbracket\right)$ is contained in either $\llbracket e \rrbracket$ or 
$\llbracket \bar{e} \rrbracket$. Therefore by Lemma 2.3 we have $\mathcal{C}_{e} \subseteq Z_{e}^{\prime}$. By Lemmas 2.3 and 3.6 we have $Z_{e}^{\prime} \subseteq \mathcal{C}_{e}$. Hence $Z_{e}^{\prime}=\mathcal{C}_{e}$.

3.2. Examples of $\mathcal{C}\left(T \times T^{\prime}\right)$. We now present some examples of computing the map on ends and building the core for some trees $T, T^{\prime} \in c v_{k}$.

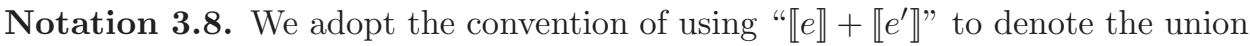

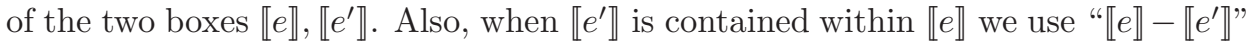
to denote the set of ends contained within $\llbracket e \rrbracket$ but not $\llbracket e^{\prime} \rrbracket$.

Example 3.9. Let $T \in c v_{2}$ and $\phi \in \operatorname{Aut}\left(F_{2}\right)$ be as in Example 3.2 We can identify $T$ with the Cayley graph for $F_{2}$ and the ends of $T$ with right-infinite words in $F_{2}$. From Figure 1 we see the map on ends $f_{\infty}: \partial T \phi \rightarrow \partial T$ is:

$$
\begin{aligned}
f_{\infty}: \llbracket a \rrbracket & \mapsto \llbracket a \rrbracket-\llbracket a B \rrbracket \\
\llbracket b \rrbracket & \mapsto \llbracket b \rrbracket-\llbracket b A B \rrbracket-\llbracket b A A \rrbracket
\end{aligned}
$$

In terms of right-infinite words, $f_{\infty}(\llbracket a \rrbracket)=\llbracket a \rrbracket-\llbracket a B \rrbracket$ is interpreted as saying that the right-infinite words starting with $a$ are taken homeomorphically by $\phi^{-1}$ to the set of right-infinite words starting with $a$ except those that start with $a B$.

Also, from Figure 1, we see that the subtrees $T_{a}^{\prime}$ and $T_{b}^{\prime}$ do not have any removable vertices, therefore the slices $\mathcal{C}_{a}, \mathcal{C}_{b}$ of the core $\mathcal{C}(T \phi \times T)$ are the respective subtrees with the "half" edges removed. Thus the slice $\mathcal{C}_{b}$ is the edge $b A$ and the slice $\mathcal{C}_{a}$ is the vertex $a \tilde{*}$. This is not the complete description of the core in this case, there are some vertical edges $(v \times e)$ that can be found by examining a homotopy inverse to $\sigma^{\prime}$. This does however give us all of the $2-$ cells in the core, hence we see $i(T \phi, T)=1$.

Example 3.10. Let $T \in c v_{3}$ be the tree with all edge lengths equal to 1 and such that $\Gamma=T / F_{3}$ is the 3 -rose whose petals are labeled $a, b, c$. In this example we will use the above algorithm to find the map on ends $f_{\infty}: \partial T \rightarrow \partial T \phi$ and build the slices of the core $\mathcal{C}(T \times T \phi)$ for the automorphism $\phi \in \operatorname{Aut}\left(F_{3}\right)$ given by $\phi(a)=$ $b a C, \phi(b)=c A, \phi(c)=a$ where $F_{3}=\langle a, b, c\rangle$, here we use capital letters to denote the inverses of the generators. The inverse of $\phi$ is the map $\phi^{-1}(a)=c, \phi^{-1}(b)=$ $a b, \phi^{-1}(c)=b c$. There is an obvious homotopy equivalence $\sigma^{\prime}: \Gamma \rightarrow \Gamma$ representing $\phi^{-1}$. Subdivide the edges of $\Gamma$ as in Example 3.2. We can assume that $\sigma^{\prime}\left(p_{a}\right)=p_{c}$. Further subdivide $b_{+}=b_{1} b_{2}$ and $b_{-}=b_{3} b_{4}$ with subdivision points $p_{b_{+}}, p_{b_{-}}$such that $\sigma^{\prime}\left(p_{b_{+}}\right)=p_{a}$ and $\sigma^{\prime}\left(p_{b_{-}}\right)=p_{b}$. Likewise subdivide both $c_{+}=c_{1} c_{2}$ and $c_{-}=c_{3} c_{4}$ with subdivision points $p_{c_{+}}, p_{c_{-}}$such that $\sigma^{\prime}\left(p_{c_{+}}\right)=p_{b}, \sigma^{\prime}\left(p_{c_{-}}\right)=p_{c}$. Our preferred paths are $\alpha_{a}=a_{+}, \alpha_{b}=b_{+}$and $\alpha_{c}=c_{+}$.

The preimages of $p_{a}, p_{b}, p_{c}$ are $\Sigma_{a}=\left\{p_{b_{+}}\right\}, \Sigma_{b}=\left\{p_{b_{-}}, p_{c_{+}}\right\}$and $\Sigma_{c}=\left\{p_{a}, p_{c_{-}}\right\}$. As $\left[\sigma^{\prime}\left(b_{1}\right)\right]=\left[a_{+}\right]=\alpha_{a}$ we see that $f_{\infty}(\llbracket a \rrbracket)=\llbracket b \rrbracket$. Then $\left[\sigma^{\prime}\left(c A B_{4}\right)\right]=\left[b c C B_{-}\right]=$ $b_{+}=\alpha_{b}$ and $\left[\sigma^{\prime}\left(c_{1}\right)\right]=\left[b_{+}\right]=\alpha_{b}$, hence $f_{\infty}(\llbracket b \rrbracket)=\llbracket c \rrbracket-\llbracket c A B \rrbracket$. Finally we see $\left[\sigma^{\prime}\left(a_{+}\right)\right]=\left[c_{+}\right]=\alpha_{c}$ and $\left[\sigma\left(a C_{4}\right)\right]=\left[c C_{-}\right]=c_{+}=\alpha_{c}$ and hence $f_{\infty}(\llbracket c \rrbracket)=$ $\llbracket a \rrbracket-\llbracket a C \rrbracket$.

$F_{k}$-equivariance can be used to find the image of any other box, for example we will compute $f_{\infty}(\llbracket B \rrbracket)$. First notice that $\llbracket B \rrbracket=B(\neg \llbracket b \rrbracket)$ where $\neg \llbracket b \rrbracket$ denotes the complement of $\llbracket b \rrbracket$ in $\partial T$. Therefore $f_{\infty}(\llbracket B \rrbracket)=f_{\infty}(B(\neg \llbracket b \rrbracket))=\phi(B) f_{\infty}(\neg \llbracket b \rrbracket)=$ $a C(\llbracket a \rrbracket+\llbracket A \rrbracket+\llbracket b \rrbracket+\llbracket B \rrbracket+\llbracket c A B \rrbracket+\llbracket C \rrbracket)=\llbracket a C \rrbracket+\llbracket B \rrbracket$.

From $f_{\infty}$ we see that the slice $\mathcal{C}_{a}$ is empty ( $a \times c$ is a twice-light rectangle), $\mathcal{C}_{c}$ is a single vertex and $\mathcal{C}_{b}$ is a single edge $c A$. Hence $i(T, T \phi)=1$. 
To get a more complicated example of the core, we look at $\mathcal{C}\left(T \times T \phi^{3}\right)$. Explicitly, $\phi^{3}$ is the automorphism:

$$
\begin{aligned}
a & \mapsto a c A B c A b a C A c A B \\
b & \mapsto b a C a c A B a C \\
c & \mapsto c A b a C A
\end{aligned}
$$

The third power of the above map $f_{\infty}$ is the map on ends for $\phi^{3}$. To find this map, we can either use the algorithm with the automorphism $\phi^{-3}$, or we can formally iterate $f_{\infty}$. For example, to find $f_{\infty}^{2}(\llbracket b \rrbracket)=f_{\infty}(\llbracket c \rrbracket-\llbracket c A B \rrbracket)=f_{\infty}(\llbracket c \rrbracket)-$ $\phi(c A) f_{\infty}(\llbracket B \rrbracket)=\llbracket a \rrbracket-\llbracket a C \rrbracket-a c A B(\llbracket a C \rrbracket+\llbracket B \rrbracket)=\llbracket a \rrbracket-\llbracket a C \rrbracket-\llbracket a c a B a C \rrbracket-$ $\llbracket a c A B B \rrbracket$. From either procedure we find:

$$
\begin{gathered}
\llbracket a \rrbracket \mapsto \llbracket a \rrbracket-\llbracket a C \rrbracket-\llbracket a c A B B \rrbracket-\llbracket a c A B a C \rrbracket \\
f_{\infty}^{3}: \llbracket b \rrbracket \mapsto \llbracket b \rrbracket-\llbracket b a C A \rrbracket-\llbracket b a C C \rrbracket-\llbracket b a C a c A B a C B \rrbracket-\llbracket b a C a c A B a C a C \rrbracket \\
\quad-\llbracket b a C a c A B a C b a C C \rrbracket-\llbracket b a C a c A B a C b a C A \rrbracket \\
\llbracket c \rrbracket \mapsto \llbracket c \rrbracket-\llbracket c A B \rrbracket-\llbracket c A b a C A C \rrbracket-\llbracket c A b a C A A \rrbracket-\llbracket c A b a C A c A B \rrbracket
\end{gathered}
$$

Using this map and Lemma 3.7 we can build the slices of the core $\mathcal{C}\left(T \times T \phi^{3}\right)$. The sets $e \times \mathcal{C}_{e}$ for $e=a, b, c$ are displayed in Figure 3. The labeling of the edges and vertices is as follows: for each set $e \times \mathcal{C}_{e}$, the bottom left vertex is shown. For instance for the set $a \times \mathcal{C}_{a}$, this vertex is $(*, a)$ where $*$ denotes the basepoint and $a$ denotes the image of the basepoint by $a$. The rest of the edges are labeled by their image in the rose $T / F_{3}$. Hence the two vertices adjacent to $(*, a)$ are $(a, a)$ and $(*, a c)$. Reading upward, the remaining vertices above $(*, a)$ are $(*, a c)$, $(*, a c A),(*, a c A B)$ and $(*, a c A B a)$. Looking at the image for $f_{\infty}(\llbracket a \rrbracket)$, we see that the span of these vertices is the slice $\mathcal{C}_{a}$. Adding up the number of squares we see $i\left(T, T \phi^{3}\right)=23$.

The colors and arrows denote the identifications, the thick black lines are free edges. As an example of the identifications let's look at the vertex on the bottom left, this vertex is $(*, a)$. Then $c(*, a)=\left(c, \phi^{3}(c) a\right)=(c, c A b a C)$, which is the fifth vertex from the bottom on the right. The identifications for the other vertices are found similarly. The observant reader will note that $\chi\left(\mathcal{C}\left(T \times T \phi^{3}\right)\right)=-2$ as expected.

\section{Bounding CONSOLIDATION}

When the morphism $f: T^{\prime} \rightarrow T$ used in Section 3 is the lift of a train-track representative, we are able to control the difference in volume between $T_{e}^{\prime}$ and $\mathcal{C}_{e}$ by bounding the amount of consolidation that occurs when removing removable vertices. Any train-track map satisfies the hypothesis used in Section 3.1 that the image of a small open neighborhood of a vertex is not contained in an edge. We begin by showing that there is a bound on the depth of consolidation.

Lemma 4.1. Let $\sigma: \Gamma \rightarrow \Gamma$ be a train-track map for a fully irreducible automorphism $\phi \in \operatorname{Out}\left(F_{k}\right)$ and $f: T \rightarrow T$ a lift. Let $e$ be an edge of $T, p_{e}$ an interior point of $e, T_{e}^{n}$ be the subtree spanned by $\left(f^{n}\right)^{-1}\left(p_{e}\right)$ and $Y_{e}^{n}$ the subtree found by iteratively removing removable vertices. There is a constant $C \geq 0$, independent of $n$, such that any subtree $A$ of $T_{e}^{n}$ that is removed in the formation of $Y_{e}^{n}$ has volume less than $C$. 


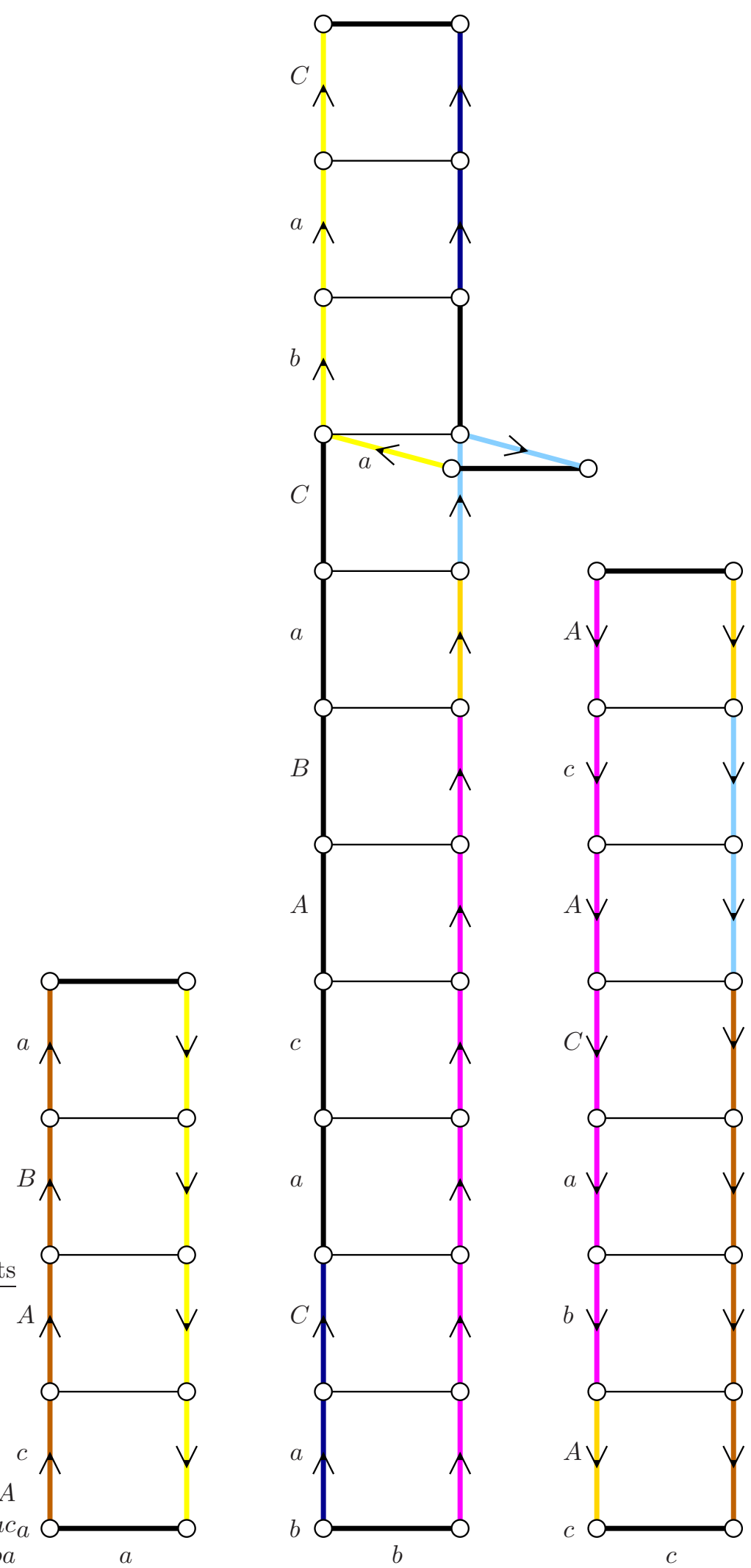

Figure 3. The core $\mathcal{C}\left(T \times T \phi^{3}\right)$ from Example 3.10 . 
Proof. Without loss of generality, we can assume that $A$ is a maximal subtree that is removed. We will show that the diameter of $A$ is bounded; since the length of an edge in $T$ is bounded and the valence of any vertex in $T$ is bounded, this implies that the volume of $A$ is bounded.

For any interior vertex $v \in A$, label the edges incident to $v$ by $\hat{e}, e_{1}, \ldots, e_{k}$ where $\hat{e}$ is the edge that leads to the unique terminal vertex of $A$ that is not a terminal vertex of $T_{e}^{n}$. Any geodesic ray originating at $v$ and containing one of the $e_{i}$ contains exactly one point from $\left(f^{n}\right)^{-1}\left(p_{e}\right)$.

We claim that the only legal turns are contained in the edge paths $\bar{e}_{i} \hat{e}$ for $i=$ $1, \ldots, k$. A turn contained in $\bar{e}_{i} e_{j}$ is illegal since $f^{n}$ identifies the germs of the edges $e_{i}$ and $e_{j}$. Since every edge must be in some legal turn we see that the turns in $\bar{e}_{i} \hat{e}$ are legal.

Therefore, the geodesic containing any two terminal vertices of $A$ has a single illegal turn. Write this geodesic as $\beta \cdot \gamma$ where $\beta, \gamma$ are legal paths. If the length of $\beta \cdot \gamma$ is more than twice the critical constant for $f$, then at least one of $\beta$ or $\gamma$ has length bounded below by the critical constant. Therefore the length of $\left[f^{m}(\beta \cdot \gamma)\right]$ goes to infinity. But since $\left[f^{m}(\beta \cdot \gamma)\right]$ is a point for $m \geq n$, we see that the diameter of $A$ is bounded by twice the critical constant.

Proposition 4.2. Let $\sigma: \Gamma \rightarrow \Gamma$ be a train-track map for a fully irreducible automorphism $\phi \in \operatorname{Out}\left(F_{k}\right)$ and $f: T \rightarrow T$ a lift. Let $e$ be an edge of $T, p_{e}$ an interior point of e, $T_{e}^{n}$ the subtree spanned by $\left(f^{n}\right)^{-1}\left(p_{e}\right)$ and $\mathcal{C}_{e}^{n}$ the slice of the core $\mathcal{C}\left(T \times T \phi^{n}\right)$ above $e$. Then there exist constants $K \geq 1$ and $C \geq 0$ such that for any $n \geq 0$ :

$$
\operatorname{vol}\left(\mathcal{C}_{e}^{n}\right) \leq \operatorname{vol}\left(T_{e}^{n}\right) \leq K \operatorname{vol}\left(\mathcal{C}_{e}^{n}\right)+C
$$

Proof. As before, denote by $Y_{e}^{n}$ the subtree of $T_{e}^{n}$ obtained by iteratively removing removable vertices and $Z_{e}^{n}$ the subtree of interior edges of $Y_{e}^{n}$. Then by Lemma 2.3 we have that $Z_{e}^{n}=\mathcal{C}_{e}^{n}$. Let $M$ and $m$ denote the length of the longest and shortest edges of $\Gamma$ respectively and let $b$ denote the maximum valence of any vertex in $\Gamma$. Then by adding at most $b$ edges of length $M$ to each vertex of $Z_{e}^{n}$, we can cover $Y_{e}^{n}$. Since $Z_{e}^{n}$ has at most $\frac{\operatorname{vol}\left(Z_{e}^{n}\right)}{m}+1$ vertices, this shows that there are constants $K_{1} \geq 1$ and $C_{1} \geq 0$ such that:

$$
\operatorname{vol}\left(\mathcal{C}_{e}^{n}\right) \leq \operatorname{vol}\left(Y_{e}^{n}\right) \leq K_{1} \operatorname{vol}\left(\mathcal{C}_{e}^{n}\right)+C_{1} .
$$

Therefore we only need to show that we can find constants $K_{2}, C_{2}$ such that $\operatorname{vol}\left(T_{e}^{n}\right) \leq K_{2} \operatorname{vol}\left(Y_{e}^{n}\right)+C_{2}$.

By Lemma 4.1 $Y_{e}^{n}$ is obtained from $T_{e}^{n}$ by removing disjoint subtrees, all of whose volumes are bounded by a constant $K_{3}$. Since the maximum number of these trees is $b\left(\frac{\operatorname{vol}\left(Y_{e}^{n}\right)}{m}+1\right)$ we see that:

$$
\operatorname{vol}\left(T_{e}^{n}\right) \leq \operatorname{vol}\left(Y_{e}^{n}\right)+K_{3} b\left(\frac{\operatorname{vol}\left(Y_{e}^{n}\right)}{m}+1\right)=\left(\frac{K_{3} b}{m}+1\right) \operatorname{vol}\left(Y_{e}^{n}\right)+K_{3} b .
$$

Hence the proposition follows.

Combining the above results, we get a way to estimate the growth rate of the intersection number for a fully irreducible automorphism.

Proposition 4.3. Let $\phi$ be a fully irreducible automorphism and $f: T \rightarrow T$ a lift of a train-track representative for $\phi$. Then for any edge $e \subset T$ and trees $T^{\prime}, T^{\prime \prime} \in c v_{k}$ :

$$
i\left(T^{\prime}, T^{\prime \prime} \phi^{n}\right) \sim \operatorname{vol}\left(T_{e}^{n}\right)
$$


where $p_{e}$ is the midpoint of $e$ and $T_{e}^{n} \subset T$ is the subtree spanned by the points in $\left(f^{n}\right)^{-1}\left(p_{e}\right)$.

Proof. By Proposition 4.2 we have $\operatorname{vol}\left(T_{e}^{n}\right) \sim \operatorname{vol}\left(\mathcal{C}_{e}^{n}\right)$ where $\mathcal{C}_{e}^{n}$ is the slice of $\mathcal{C}\left(T \times T \phi^{n}\right)$ above $e$. Since $\phi$ is fully irreducible, $\operatorname{vol}\left(T_{e}^{n}\right) \sim \operatorname{vol}\left(T_{e^{\prime}}^{n}\right)$ for any edges $e, e^{\prime} \subset T$. (This becomes clear in Section 5 where we compute $\operatorname{vol}\left(T_{e}^{n}\right)$ up to $\sim$ equivalence independent of the edge $e$.) Thus $\operatorname{vol}\left(T_{e}^{n}\right) \sim \sum_{e^{\prime} \subset T / F_{k}} l_{T}\left(e^{\prime}\right) \operatorname{vol}\left(\mathcal{C}_{e^{\prime}}^{n}\right)=$ $i\left(T, T \phi^{n}\right) \sim i\left(T^{\prime}, T^{\prime \prime} \phi^{n}\right)$. The final equivalence is from Proposition 2.9.

Using the techniques developed so far, we can get a lower bound on the asymptotics of $n \mapsto i\left(T, T^{\prime} \phi^{n}\right)$.

Corollary 4.4. Suppose $\phi$ is a fully irreducible automorphism and let $\lambda$ and $\mu$ denote the growth rates of $\phi$ and $\phi^{-1}$ respectively. Then for any $T, T^{\prime} \in c v_{k}$, there exist constants $K \geq 1$ and $C \geq 0$ such that $\frac{1}{K} \max \{\lambda, \mu\}^{n}-C \leq i\left(T, T^{\prime} \phi^{n}\right)$.

Proof. By Proposition 4.3 we only need to find a lower bound for $\operatorname{vol}\left(T_{e}^{n}\right)$ where

$T_{e}^{n}$ is the span of $\left(f^{n}\right)^{-1}\left(p_{e}\right)$ for a lift of a train-track map $f: T \rightarrow T$. Since $\phi$ is fully irreducible and $f$ uniformly expands edges by $\lambda$, the cardinality of $\left(f^{n}\right)^{-1}\left(p_{e}\right)$ is $\sim \lambda^{n}$. Since each point of $\left(f^{n}\right)^{-1}\left(p_{e}\right)$ lies in a unique edge of $T_{e}^{n}$, there exists constants $K \geq 1$ and $C \geq 0$ such that $\frac{1}{K} \lambda^{n}-C \leq i\left(T, T \phi^{n}\right)$. Repeating this argument for $f^{\prime}: T^{\prime} \rightarrow T^{\prime}$, a lift of a train-track map representing $\phi^{-1}$, we see that there are constants $K^{\prime} \geq 1$ and $C^{\prime} \geq 0$ such that $\frac{1}{K^{\prime}} \mu^{n}-C^{\prime} \leq i\left(T^{\prime}, T^{\prime} \phi^{-n}\right)$. Since $i\left(T^{\prime}, T^{\prime} \phi^{n}\right) \sim i\left(T, T \phi^{-n}\right)$, the result follows.

\section{Growth Rates}

In this section we compute the growth rate of $n \mapsto \operatorname{vol}\left(T_{e}^{n}\right)$ where $T_{e}^{n}$ is as in Proposition 4.3. There are two cases depending on whether or not $T^{+}$, the stable tree of $\phi$, is geometric. Every fully irreducible automorphism admits a train-track representative (called stable) with at most one orbit of indivisible Nielsen paths (this was proved in [4, Lemma 3.2] in the case of period 1 but the proof in general is identical).

Moreover the existence of an indivisible Nielsen path characterizes whether $T^{+}$ is geometric.

Theorem 5.1 (Theorem 3.2 [2]). Let $\phi \in \operatorname{Out}\left(F_{k}\right)$ be a fully irreducible automorphism, $\sigma: \Gamma \rightarrow \Gamma$ a stable train-track map and $T^{+}$the stable tree for $\phi$. Then $T^{+}$ is geometric if and only if $\sigma: \Gamma \rightarrow \Gamma$ contains an indivisible orbit of Nielsen paths.

We will not make use any of the additional properties that stableness of a traintrack map guarantees with the exception of Proposition 5.20. These properties are mentioned within this proposition. See 4 for a definition. The following definition is of central importance to understanding the tree $T_{e}^{n}$ from Proposition 4.3 .

Definition 5.2. An i-vanishing path for $\sigma: \Gamma \rightarrow \Gamma(i \geq 0)$ is an immersion $\iota:[0,1] \rightarrow \Gamma$ such that the image $\sigma^{i} \iota([0,1])$ is homotopic to a point relative to the endpoints. We will always assume that $i$ is minimal. If $f: T \rightarrow T$ is a lift of $\sigma: \Gamma \rightarrow \Gamma$, a $i$-vanishing path is an embedding $\iota:[0,1] \rightarrow T$ such that $f^{i} \iota(0)=f^{i} \iota(1)$. Clearly any $i$-vanishing path in $T$ projects to a $i$-vanishing path in $\Gamma$ and vice versa. A vanishing path is an $i$-vanishing path for some $i$.

The importance of vanishing paths is given by the following remark. 
Remark 5.3. Suppose $\sigma: \Gamma \rightarrow \Gamma$ is a map and $f: T \rightarrow T$ is a lift. Let $T_{e}^{n}$ be the subtree spanned by $\left(f^{n}\right)^{-1}\left(p_{e}\right)$ for any edge $e \subset T$. Then $T_{e}^{n}$ can be expressed as a union of $i$-vanishing paths where $i \leq n$. Infact, the path joining any two points in $\left(f^{n}\right)^{-1}\left(p_{e}\right)$ is an $i$-vanishing path for some $i \leq n$.

Therefore we are interested in examining the lengths of vanishing path. As we demonstrate in Section 5.2. every indivisible Nielsen path $\gamma$ contains subpaths $\gamma_{\epsilon}$ that are $i$-vanishing, for arbitrarily large $i$. Note that all such vanishing paths have uniformly bounded length. In Section 5.1 we will prove a converse to this observation in the absence of indivisible Nielsen paths. Specifically, we show that:

1. If $T^{+}$is geometric then every vanishing path is a composition of suitable $\gamma_{\epsilon}$ 's. See Proposition 5.22

2. If $T^{+}$is nongeometric then there exist arbitrarily long vanishing paths that are not compositions of shorter vanishing paths. In fact, any $i$-vanishing path has length approximately $\mu^{i}$, where $\mu$ is the expansion factor for $\phi^{-1}$. See Proposition 5.15 .

Example 5.24 shows these different behaviors. We begin by examining the case when $T^{+}$is nongeometric.

\section{1. $T^{+}$nongeometric.}

Convention 5.4. We fix some notation for use in the rest of this section: $\phi$ is a fully irreducible automorphism, $\sigma: \Gamma \rightarrow \Gamma$ is a train-track map for $\phi$ with expansion factor $\lambda, \sigma^{\prime}: \Gamma^{\prime} \rightarrow \Gamma^{\prime}$ is a train-track map for $\phi^{-1}$ with expansion factor $\mu$ and $\tau: \Gamma \rightarrow \Gamma^{\prime}, \tau^{\prime}: \Gamma^{\prime} \rightarrow \Gamma$ are Lipschitz homotopy equivalences representing the change in marking. This is summarized in the following commutative (up to homotopy) diagram:

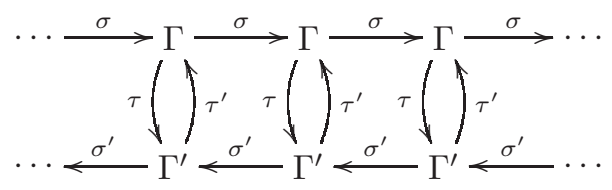

As stated above, we will show that when $\Gamma$ does not contain an indivisible orbit of periodic Nielsen paths that the length of an $i$-vanishing path for $\sigma$ is approximately $\mu^{i}$. First we give an upper bound on the length of an $i$-vanishing path. The following proposition does not depend on the absence of an indivisible orbit of periodic Nielsen paths.

Proposition 5.5. Let $\sigma: \Gamma \rightarrow \Gamma$ be as in Convention 5.4 There exists a constant $K \geq 0$ such that for every $i$-vanishing path $\gamma$ for $\sigma: \Gamma \rightarrow \Gamma$ we have length $(\gamma) \leq$ $K \mu^{i}$.

Proof. Since the composition $\sigma^{\prime} \tau \sigma$ is homotopic to $\tau$ and the composition $\tau^{\prime} \tau$ is homotopic to the identity map, there are constants $K_{1}, K_{2} \geq 1$, such that any path $\gamma$ in $\Gamma$ satisfies

$$
\begin{aligned}
\mid \text { length }([\tau(\gamma)])-\operatorname{length}\left(\left[\sigma^{\prime} \tau \sigma(\gamma)\right]\right) \mid & \leq K_{1} \text { and } \\
\left|\operatorname{length}(\gamma)-\operatorname{length}\left(\left[\tau^{\prime} \tau(\gamma)\right]\right)\right| & \leq K_{2} .
\end{aligned}
$$

For a 1-vanishing path $\gamma$, using the inequality (3) we have

$$
\text { length }([\tau(\gamma)]) \leq \text { length }\left(\left[\sigma^{\prime} \tau \sigma(\gamma)\right]\right)+K_{1}=K_{1}
$$


as length $([\sigma(\gamma)])=0$.

We now proceed by induction. Suppose that the image under $\tau$ of any $(i-1)-$ vanishing path has length at most $K_{1} \sum_{j=1}^{i-1} \mu^{j-1}$; note that the previous paragraph verified the base case, a 1-vanishing path. Now consider an $i$-vanishing path $\gamma$. Then $\sigma(\gamma)$ is an $(i-1)$-vanishing path, and thus the inductive hypothesis yields length $([\tau \sigma(\gamma)]) \leq K_{1} \sum_{j=1}^{i-1} \mu^{j-1}$. Since $\sigma^{\prime}$ is a train-track map this implies length $\left(\left[\sigma^{\prime} \tau \sigma(\gamma)\right]\right) \leq \mu K_{1} \sum_{j=1}^{i-1} \mu^{j-1}$. Then (3) implies length $([\tau(\gamma)]) \leq$ length $\left(\left[\sigma^{\prime} \tau \sigma(\gamma)\right]\right)+K_{1}$, thus yielding the following which completes our induction:

$$
\text { length }([\tau(\gamma)]) \leq \mu K_{1} \sum_{j=1}^{i-1} \mu^{j-1}+K_{1}=K_{1} \sum_{j=1}^{i} \mu^{j-1} .
$$

Therefore $\operatorname{length}\left(\left[\tau^{\prime} \tau(\gamma)\right]\right) \leq K_{3} K_{1} \sum_{j=1}^{i} \mu^{j-1}$ where $K_{3}$ is the Lipschitz constant for $\tau^{\prime}$. As before, using (44) we have

$$
\text { length }(\gamma) \leq K_{3} K_{1} \sum_{j=1}^{i} \mu^{j-1}+K_{2} .
$$

Setting $K=K_{3} K_{1} \frac{1}{\mu-1}+K_{2}$ completes the proof.

Using Proposition 5.5 we can estimate the difference between the homotopic maps $\tau$ and $\sigma^{\prime n} \tau \sigma^{n}$.

Lemma 5.6. Let $\sigma: \Gamma \rightarrow \Gamma$ be as in Convention 5.4. Then there is a a constant $K \geq 0$ such that for any path $\gamma \subset \Gamma$ and $n \geq 0$ we have

$$
\text { length }([\tau(\gamma)]) \geq \operatorname{length}\left(\left[\sigma^{\prime n} \tau \sigma^{n}(\gamma)\right]\right)-K \mu^{n} .
$$

Proof. Since $\tau$ is homotopic to $\sigma^{\prime n} \tau \sigma^{n}$, if $\gamma$ is a closed loop then length $([\tau(\gamma)])=$ length $\left(\left[\sigma^{\prime n} \tau \sigma^{n}(\gamma)\right]\right)$.

If $\gamma$ is not a loop, we can add a segment $\alpha$ of bounded length to $\left[\sigma^{n}(\gamma)\right]$ to get a closed loop $\gamma_{1}$ such that $\left[\sigma^{n}(\gamma)\right]$ is a subpath of $\gamma_{1}$ and $\alpha$ is an embedded arc. Let $\gamma_{0}$ be a closed loop in $\Gamma$ such that $\left[\sigma^{n}\left(\gamma_{0}\right)\right]=\gamma_{1}$. As $\alpha$ has bounded length, there is a constant $K_{1}$ such that length $\left(\left[\sigma^{\prime n} \tau(\alpha)\right]\right) \leq K_{1} \mu^{n}$. Hence:

$$
\begin{aligned}
\operatorname{length}\left(\left[\tau\left(\gamma_{0}\right)\right]\right) & =\operatorname{length}\left(\left[\sigma^{\prime n} \tau\left(\gamma_{1}\right)\right]\right) \\
& \geq \operatorname{length}\left(\left[\sigma^{\prime n} \tau \sigma^{n}(\gamma)\right]\right)-\operatorname{length}\left(\left[\sigma^{\prime n} \tau(\alpha)\right]\right) \\
& \geq \operatorname{length}\left(\left[\sigma^{\prime n} \tau \sigma^{n}(\gamma)\right]\right)-K_{1} \mu^{n} .
\end{aligned}
$$

There is path a $\beta$ in $\Gamma$ (unique up to homotopy) such that the concatenation of $\gamma$ and $\beta$ is homotopic to $\gamma_{0}$. Notice that $\left[\sigma^{n}(\beta)\right]=\alpha$ as $\left[\sigma^{n}(\gamma)\right]$ is a subpath of $\gamma_{1}$. Now length $([\tau(\gamma)]) \geq$ length $\left(\left[\tau\left(\gamma_{0}\right)\right]\right)-$ length $([\tau(\beta)])$. We will show that $\beta$ is the union of a bounded number of $i$-vanishing paths where $i \leq n$ along with some segments of bounded length.

Let $x$ and $y$ be the endpoints of $\alpha$ and $p$ and $q$ the endpoints of $\beta$ with $\sigma^{n}(p)=x$ and $\sigma^{n}(q)=y$. Now decompose $\beta=\beta_{0} \cdot \beta_{1}$ by subdividing $\beta$ at the point in $\left(\sigma^{n}\right)^{-1}(y)$ that is closest (along $\beta$ ) to $x$. Then $\beta_{1}$ is an $i$-vanishing path for $\sigma$ with $i \leq n$ as $\alpha$ is embedded and $\left[\sigma^{n}(\beta)\right]=\alpha$. Thus $\left[\sigma^{n}\left(\beta_{0}\right)\right]=\alpha$. Similarly, decompose $\beta_{0}=\beta_{2} \cdot \beta_{3}$ where $\beta_{2}$ is an $i^{\prime}$-vanishing path for $\sigma$ with $i^{\prime} \leq n$ and where $\left[\sigma^{n}\left(\beta_{2}\right)\right]=x$. Thus $\left[\sigma^{n}\left(\beta_{3}\right)\right]=\alpha$ and $\left(\sigma^{n}\right)^{-1}(x)$ and $\left(\sigma^{n}\right)^{-1}(y)$ only intersect $\beta_{3}$ in its endpoints. Now repeat at the vertices contained in $\alpha$ to decompose $\beta_{3}$ as a union 
of vanishing paths (the number of which is bounded by the number of vertices of $\Gamma)$ connected by segments that are homeomorphically mapped to the edges of $\alpha$. The length and number of such segments is bounded. Hence by Proposition 5.5 and since $\tau$ induces a Lipschitz map between the universal covers of $\Gamma$ and $\Gamma^{\prime}$ there is a constant $K_{2}$ such that length $([\tau(\beta)]) \leq K_{2} \mu^{n}$. Setting $K=K_{1}+K_{2}$ completes the proof.

To show the correct lower bound on the length of an $i$-vanishing path for $\sigma: \Gamma \rightarrow$ $\Gamma$ we need the following lemma from $[3$.

Lemma 5.7 (Lemma 2.9 [3]). Let $\sigma: \Gamma \rightarrow \Gamma$ be a train-track map representing a fully irreducible outer automorphism $\phi$. Then for every $C>0$ there is a number $M>0$ such that if $\gamma$ is any path, then one of the following holds:

1. $\left[\sigma^{M}(\gamma)\right]$ contains a legal segment of length $>C$.

2. $\left[\sigma^{M}(\gamma)\right]$ has fewer illegal turns than $\gamma$.

3. $\gamma$ is a concatenation $x \cdot y \cdot z$ with $\left[\sigma^{M}(y)\right]$ (periodic) Nielsen and $x$ and $z$ have length $\leq 2 C$ and at most one illegal turn.

Using the above we can show that when $\Gamma$ does not contain an orbit of periodic Nielsen paths then short vanishing paths for $\sigma$ vanish quickly. First we need to understand paths in $\Gamma$ that could satisfy conclusion 3 in Lemma 5.7

Lemma 5.8. Let $\sigma: \Gamma \rightarrow \Gamma$ be as in Convention 5.4 and suppose that $\Gamma$ does not contain an indivisible orbit of periodic Nielsen paths. Then there exists an $N>0$ such that if $\gamma$ is an $i$-vanishing path for $\sigma: \Gamma \rightarrow \Gamma$ with at most 2 illegal turns, then $i \leq N$.

Proof. Suppose otherwise, therefore we have sequence of $i_{j}$-vanishing paths $\gamma_{j}$ with at most 2 illegal turns where $i_{j}<i_{j+1}$. We will show that this implies that some power of $\sigma$ has a Nielsen path which contradicts our assumption.

First note that the lengths of $\gamma_{j}$ are uniformly bounded, since legal subpaths of vanishing paths have length bounded by the critical constant. By passing to a subsequence we can assume that the paths $\gamma_{j}$ all have the same combinatorial type, i.e, they cross the same turns of $\Gamma$ in the same order. Further, by passing to a power of $\sigma$, possibly replacing the sequence $\gamma_{j}$ by $\sigma^{\ell}\left(\gamma_{j}\right)$ for some $\ell$ such that the vertex (or vertices) at the illegal turn is (are) fixed by $\sigma$ and again passing to a subsequence, we can assume that $\left[\sigma\left(\gamma_{j}\right)\right] \subseteq \gamma_{j}$.

We will break the proof up into two cases depending on whether the paths $\gamma_{j}$ contain a single illegal turn or two illegal turns. First assume that the paths $\gamma_{j}$ only contain a single illegal turn. Notice that any $i$-vanishing path with a single illegal turn has the form $a \cdot b$ where $a$ and $b$ are legal segments of the same length such that $\sigma^{i}(a)=\sigma^{i}(\bar{b})$. Further any subpath of the form $a^{\prime} \cdot b^{\prime}$ where $a^{\prime}$ and $b^{\prime}$ are legal segments of the same length is an $i^{\prime}$-vanishing path where $i^{\prime} \leq i$.

We have two claims about the vanishing paths $\gamma_{j}$.

Claim 1: If $\iota$ is a subpath of $\gamma_{j}$ and $\iota$ is an $i$-vanishing path for $\sigma: \Gamma \rightarrow \Gamma$ then $i \leq i_{j}$.

Proof of Claim. If $\left[\sigma^{i_{j}}(\iota)\right]$ is not a point, then it is a path that does not contain any illegal turns (as there is a single illegal turn in $\gamma_{j}$ ) and hence $\iota$ is not a vanishing path. 
Claim 2: If $j<k$ then $\gamma_{j}$ is a subpath of $\gamma_{k}$.

Proof of Claim. Since the two legal segments in each of $\gamma_{j}$ and $\gamma_{k}$ have equal lengths, we must have $\gamma_{j} \subset \gamma_{k}$ or $\gamma_{k} \subset \gamma_{j}$ because they have the same combinatorial type. The latter is not possible by Claim 1 .

Therefore by the above Claims, we have $\gamma_{j} \subset \gamma_{j+1}$ and hence there is a welldefined limit $\gamma_{\infty}$. By construction, we have $\gamma_{\infty}=a \cdot b$ where $a$ and $b$ are legal segments of the same length as this holds for each of the $\gamma_{j}$. Further $[\sigma(a)]$ and $[\sigma(b)]$ are also legal segments of the same length and $\left[\sigma\left(\gamma_{\infty}\right)\right] \subseteq\left[\gamma_{\infty}\right]$. If $\left[\sigma\left(\gamma_{\infty}\right)\right] \neq \gamma_{\infty}$ then $\left[\sigma\left(\gamma_{\infty}\right)\right] \subset \gamma_{j^{\prime}}$ for some $j^{\prime}$ and as $[\sigma(a)]$ and $[\sigma(b)]$ are also legal segments of the same length, $\left[\sigma\left(\gamma_{\infty}\right)\right]$ is a $i^{\prime}$-vanishing path for some $i^{\prime} \leq i$. However, since $\gamma_{j} \subset \gamma_{\infty}$ are all $i_{j}$-vanishing paths with $i_{j} \rightarrow \infty$, by Claim 1 , the path $\gamma_{\infty}$ is not a vanishing path. Therefore $\left[\sigma\left(\gamma_{\infty}\right)\right]=\gamma_{\infty}$ and hence $\gamma_{\infty}$ is a Nielsen path.

It remains to consider the case when $\gamma_{j}$ 's have two illegal turns. We will argue that this is not possible. Suppose $\gamma$ is a vanishing path with two illegal turns and $[\sigma(\gamma)] \subset \gamma$. The middle legal segment $b$ of $\gamma=a \cdot b \cdot c$ maps over itself and therefore has a unique fixed point, breaking it up as $b=b_{1} b_{2}$. There are two subcases depending on whether $b$ maps over itself preserving or reversing the orientation; the cases are similar and we assume the orientation is preserved. Therefore the iterates of $a \cdot b_{1}$ and of $b_{2} \cdot c$ never cancel against each other, so both must be vanishing. But $\left[\sigma\left(a \cdot b_{1}\right)\right]$ has the form $a^{\prime} \cdot b_{1}$ for some $a^{\prime} \subset a$ (and both $a b_{1}$ and $a^{\prime} b_{1}$ are vanishing). This contradicts the fact that vanishing paths with one illegal turn have legal segments of equal lengths.

This completes the proof of the lemma.

Lemma 5.9. Let $\sigma: \Gamma \rightarrow \Gamma$ be as in Convention 5.4 and suppose that $\Gamma$ does not contain an indivisible orbit of periodic Nielsen paths. For every $L>0$ there exists an $N>0$, such that if $\gamma$ is an $i$-vanishing path for $\sigma$ and length $(\gamma) \leq L$ then $i \leq N$.

Proof. As there is a lower bound on the distance between any two illegal turns in $\Gamma$, for any vanishing path with length less than $L$, the number of illegal turns in a path of length at most $L$ is at most some constant $I$.

Let $C$ be larger than the critical constant for $\sigma$ and $M$ the constant from Lemma 5.7. If $\left[\sigma^{M}(\gamma)\right]$ had a legal segment of length greater than $C$, this would imply that $\left[\sigma^{n}(\gamma)\right]$ had positive length for all $n>M$, and thus $\gamma$ is not a vanishing path, contrary to hypothesis. Thus possibility 1 in Lemma 5.7 cannot occur for any vanishing path. On the other hand, if possibility 3 of Lemma 5.7 occurs then $y$ is trivial since by assumption $\Gamma$ does not contain an indivisible orbit of periodic Nielsen paths, hence $\gamma=x \cdot z$ and has length less than $2 C$ and at most 2 illegal turns.

Thus the possibility 2 of Lemma 5.7 must occur for every vanishing path with more than 2 illegal turns or length greater than $2 C$, namely $\left[\sigma^{M}(\gamma)\right]$ has strictly fewer illegal turns than $\gamma$. Hence $\left[\sigma^{I M}(\gamma)\right]$ has at most two illegal turns and length less than $2 C$ for any vanishing path $\gamma$. Therefore, we see that $\left[\sigma^{N_{0}+I M}(\gamma)\right]$ is a point, where $N$ is the constant from Lemma 5.8 and hence $i \leq N+I M$. 
To derive the more precise lower bound on the length of an $i$-vanishing path, we consider two types of legality. For a path $\gamma$ with $I$ illegal turns, we define the ratio:

$$
L E G_{1}(\gamma)=\frac{\text { length }(\gamma)-2 \lambda^{-1} B I}{\text { length }(\gamma)}
$$

where $B=B C C(\sigma)$ is the bounded cancellation constant. Since length $([\sigma(\gamma)]) \geq$ $\lambda$ length $(\gamma)-2 B I$ we see that length $([\sigma(\gamma)]) \geq \lambda L E G_{1}(\gamma)$ length $(\gamma)$. The following Lemma and Corollary do not depend on the absence of an orbit of periodic Nielsen paths.

Lemma 5.10. Let $\sigma: \Gamma \rightarrow \Gamma$ be as in Convention 5.4. For any path $\gamma \subset \Gamma$ with $L E G_{1}(\gamma)>\lambda^{-1}$, the ratio $L E G_{1}\left(\left[\sigma^{n}(\gamma)\right]\right)$ converges to 1 . Moreover, for any $0<\epsilon<1-\lambda^{-1}$, there is a $\delta>0$ such that if $L E G_{1}(\gamma) \geq \lambda^{-1}+\epsilon$ then the infinite product $\prod_{n=0}^{\infty} L E G_{1}\left(\left[\sigma^{n}(\gamma)\right]\right)$ converges to a positive number greater than or equal to $\delta$.

Proof. Since the number of illegal turns in $[\sigma(\gamma)]$ is at most the number of illegal turns in $\gamma$ and length $([\sigma(\gamma)]) \geq \lambda$ length $(\gamma)-2 B I$ we see:

$$
\begin{aligned}
\frac{1-L E G_{1}([\sigma(\gamma)])}{1-L E G_{1}(\gamma)} & \leq \frac{2 \lambda^{-1} B I}{\lambda \text { length }(\gamma)-2 B I}\left(\frac{2 \lambda^{-1} B I}{\text { length }(\gamma)}\right)^{-1} \\
& =\frac{\operatorname{length}(\gamma)}{\lambda \operatorname{length}(\gamma)-2 B I}=\frac{1}{\lambda L E G_{1}(\gamma)}<1
\end{aligned}
$$

Hence $L E G_{1}([\sigma(\gamma)])>L E G_{1}(\gamma)>\lambda^{-1}$ and so repeating the above we see that $L E G_{1}\left(\left[\sigma^{n}(\gamma)\right]\right)$ converges to 1 . By bounding $L E G_{1}(\gamma)$ away from $\lambda^{-1}$ we can make the rate of convergence independent of the path $\gamma$. Further the above calculation shows:

$$
\limsup _{n \rightarrow \infty} \frac{1-L E G_{1}\left(\left[\sigma^{n+1}(\gamma)\right]\right)}{1-L E G_{1}\left(\left[\sigma^{n}(\gamma)\right]\right)} \leq \lambda^{-1} .
$$

Hence the product $\prod_{n=1}^{\infty} L E G_{1}\left(\left[\sigma^{n}(\gamma)\right]\right)$ converges to a positive number, which can be bounded away from 0 independent of the path $\gamma$ by bounding $L E G_{1}(\gamma)$ away from $\lambda^{-1}$.

The following corollary follows immediately.

Corollary 5.11. Let $\sigma: \Gamma \rightarrow \Gamma$ be as in Convention 5.4. For any $0<\epsilon<1-\lambda^{-1}$, there is a constant $K>0$ such that for any $n \geq 0$ and any path $\gamma \subset \Gamma$ with $L E G_{1}(\gamma) \geq \lambda^{-1}+\epsilon$ :

$$
\text { length }\left(\left[\sigma^{n}(\gamma)\right]\right) \geq K \lambda^{n} \text { length }(\gamma)
$$

As a word of caution, we will be applying this corollary to paths in $\Gamma^{\prime}$ and the train-track map $\sigma^{\prime}: \Gamma^{\prime} \rightarrow \Gamma^{\prime}$. Specifically, this corollary enables us to get a lower bound on the length of a path $\gamma \subset \Gamma$ where $[\tau(\gamma)] \subset \Gamma^{\prime}$ has a large legality with respect to $\sigma^{\prime}: \Gamma^{\prime} \rightarrow \Gamma^{\prime}$.

Lemma 5.12. Let $\sigma: \Gamma \rightarrow \Gamma$ be as in Convention 5.4. For any $0<\epsilon<1-\mu^{-1}$, there exist constants $K>0$ and $C, C^{\prime} \geq 0$ such that for any $n \geq 0$ and any path $\gamma \subset \Gamma$, if $L E G_{1}\left(\left[\tau \sigma^{n}(\gamma)\right]\right) \geq \mu^{-1}+\epsilon$ then:

$$
\text { length }(\gamma) \geq K \mu^{n}\left(\text { length }\left(\left[\tau \sigma^{n}(\gamma)\right]\right)-C\right)-C^{\prime} .
$$


Proof. Applying Corollary [5.11 to $\sigma^{\prime}: \Gamma^{\prime} \rightarrow \Gamma^{\prime}$ we see that there is a constant $K_{1}>0$ such that for any path $\gamma \subset \Gamma$ with $L E G_{1}\left(\left[\tau \sigma^{n}(\gamma)\right]\right) \geq \lambda^{-1}+\epsilon$ :

$$
\text { length }\left(\left[\sigma^{\prime n} \tau \sigma^{n}(\gamma)\right]\right) \geq K_{1} \mu^{n} \text { length }\left(\left[\tau \sigma^{n}(\gamma)\right]\right) .
$$

Combining this with Lemma 5.6 there is a constant $C \geq 0$ such that:

$$
\text { length }([\tau(\gamma)]) \geq K_{1} \mu^{n}\left(\text { length }\left(\left[\tau \sigma^{n}(\gamma)\right]\right)-C\right) .
$$

Finally, since $\tau$ induces a quasi-isometry between the universal covers of $\Gamma$ and $\Gamma^{\prime}$, there are constants $K>0$ and,$C^{\prime} \geq 0$ such that

$$
\text { length }(\gamma) \geq K \mu^{n}\left(\text { length }\left(\left[\tau \sigma^{n}(\gamma)\right]\right)-C\right)-C^{\prime}
$$

We now show that if $\gamma$ is an $i$-vanishing path for $\sigma$ then $L E G_{1}(\tau(\gamma))$ can be made close to 1 for large enough $i$, thus enabling us to use Lemma 5.12. To show this we need to use the version of legality from [3]. Let $C$ be larger than critical constant for $\sigma$ and the critical constant for $\sigma^{\prime}$ and define:

$$
L E G_{2}(\gamma)=\frac{\text { sum of the lengths of the legal segments of } \gamma \text { of length } \geq C}{\text { length }(\gamma)}
$$

There is a constant $\eta$ such that, length $\left(\left[\sigma^{n}(\gamma)\right]\right) \geq \eta \lambda^{n} L E G_{2}(\gamma) \operatorname{length}(\gamma)$ for any path $\gamma$. We need the following lemma regarding this version of legality.

Lemma 5.13 (Lemma 5.6 [3]). Assume that $\phi$ has no nontrivial periodic conjugacy classes (or equivalently that $\phi$ is not represented by a homeomorphism of a punctured surface). Then there are constants $L, N, \epsilon>0$ such that for any path $\gamma$ with length $(\gamma) \geq L$ and every $n \geq N$ either $L E G_{2}\left(\sigma^{n}(\gamma)\right) \geq \epsilon$ or $L E G_{2}\left(\sigma^{\prime n} \tau(\gamma)\right) \geq \epsilon$.

In [3], the above lemma is stated for conjugacy classes in $F_{k}$ but the proof applies equally well to this setting.

Lemma 5.14. Let $\sigma: \Gamma \rightarrow \Gamma$ be as in Convention 5.4 and suppose that $\Gamma$ does not contain an indivisible orbit of periodic Nielsen paths. For all $0<\delta<1$, there are constants $L$ and $N$ such that if $\gamma$ is a vanishing path for $\sigma$ with length $\geq L$ then $L E G_{1}\left(\left[\sigma^{\prime n} \tau(\gamma)\right]\right) \geq \delta$ for all $n \geq N$.

Proof. Let $L_{1}, N_{1}$ and $\epsilon$ be constants from Lemma 5.13 Since $\gamma$ is a vanishing path for $\sigma$, we have $L E G_{2}\left(\sigma^{n}(\gamma)\right)=0$ for all $n \geq 0$ and hence we must have the second conclusion from this Lemma, namely: $L E G_{2}\left(\left[{\sigma^{\prime N_{1}}} \tau(\gamma)\right]\right) \geq \epsilon$ for vanishing paths $\gamma$ with length at least $L$.

Therefore there is a constant $\eta>0$ such that for any vanishing path with length at least $L$ :

$$
\text { length }\left(\left[\sigma^{\prime n} \tau(\gamma)\right]\right) \geq \eta \epsilon \mu^{n-N_{1}} \text { length }\left(\left[\sigma^{\prime N_{1}} \tau(\gamma)\right]\right)
$$

for $n \geq N_{1}$. Further, there is a constant $I$ such that the number of illegal turns in $\sigma^{\prime n} \tau(\gamma)$ is at most Ilength $\left(\left[\sigma^{\prime N_{1}} \tau(\gamma)\right]\right.$ ) for all $n \geq N_{1}$ (since legal turns go to legal turns and the distance between illegal turns is uniformly bounded from below). Hence set $L=L_{1}$ and $N$ large enough such that $1-\frac{2 B \mu^{-1} I}{\eta \epsilon \mu^{N-N_{1}}} \geq \delta$.

Finally, we can prove that the $i$-vanishing path have length approximately $\mu^{i}$ when there are no orbits of periodic Nielsen paths. 
Proposition 5.15. Let $\sigma: \Gamma \rightarrow \Gamma$ be as in Convention 5.4 and suppose that $\Gamma$ does not contain an indivisible orbit of periodic Nielsen paths. There exists a constants $K>0$ and $C \geq 0$ such that for every $i$-vanishing path $\gamma$ for $\sigma: \Gamma \rightarrow \Gamma$ we have length $(\gamma) \geq K \mu^{i}-C$.

Proof. Fix a small $0<\epsilon<1-\mu^{-1}$, and let $L_{1}$ and $N_{1}$ be the constants from Lemma 5.14 using $\delta=\mu^{-1}+\epsilon$. Also let $K_{1}, C_{1}, C_{1}^{\prime}$ be the constants from Lemma 5.12 using $\epsilon / 2$. Let $\ell$ be large enough such that the $\tau$-image of an $\ell$-vanishing path has length at least $2 C_{1}$ and such that an $\left(\ell-N_{1}\right)$-vanishing path has length at least $L$. Such an $\ell$ exists by Lemma [5.9.

Suppose $\alpha$ is an $\ell$-vanishing path for $\sigma$. Hence $\sigma^{N_{1}}(\alpha)$ has length at least $L_{1}$ and by Lemma 5.14 .

$$
L E G_{1}\left(\left[\sigma^{N_{1}} \tau \sigma^{N_{1}}(\alpha)\right]\right) \geq \mu^{-1}+\epsilon .
$$

Since $\tau$ is homotopic to $\sigma^{\prime N_{1}} \tau \sigma^{N_{1}}$, by further requiring that $\ell$ be sufficiently large, we can guarantee that the length of an $\ell$-vanishing path is large enough such that:

$$
L E G_{1}([\tau(\alpha)]) \geq L E G_{1}\left(\left[\sigma^{\prime N_{1}} \tau \sigma^{N_{1}}(\alpha)\right]\right)-\epsilon / 2 \geq \mu^{-1}+\epsilon / 2 .
$$

For $i>\ell$ we can apply the above to $\alpha=\sigma^{i-\ell}(\gamma)$, by Lemma 5.12 we have:

$$
\text { length }(\gamma) \geq K_{1} \mu^{i-\ell}\left(\text { length }\left(\left[\tau \sigma^{i-\ell}(\gamma)\right]\right)-C_{1}\right)-C_{1}^{\prime} .
$$

Since length $\left(\left[\tau \sigma^{i-\ell}(\gamma)\right]\right) \geq 2 C_{1}$, have length $(\gamma) \geq K_{1} \mu^{i-\ell} C_{1}-C_{1}^{\prime}$.

Recall that $T_{e}^{n}$ is the span of the points in $\left(f^{n}\right)^{-1}\left(\widetilde{p_{e}}\right)$ where $f: T \rightarrow T$ is a lift of $\sigma: \Gamma \rightarrow \Gamma$ and $p_{e}$ is a lift of a point in the interior of $e$. The path joining any pair of points in $\left(f^{n}\right)^{-1}\left(p_{e}\right)$ is an $i$-vanishing path for some $i \leq n$. We define a set of equivalence relations on the set $\left(f^{n}\right)^{-1}\left(p_{e}\right)$ by $x \sim_{i} x^{\prime}$ if the path connecting them is $j$-vanishing path where $j \leq i$. Equivalently, $x \sim_{i} x^{\prime}$ if $f^{i}(x)=f^{i}\left(x^{\prime}\right)$ for $i \leq n$. Notice that if $x \sim_{j} x^{\prime}$ and $x^{\prime} \sim_{i} x^{\prime \prime}$ for $j \leq i$ then $x \sim_{i} x^{\prime \prime}$. An $\sim_{i}$ equivalence class is called an $i$-clump. Notice that the entire set $\left(f^{n}\right)^{-1}\left(p_{e}\right)$ is an $n$-clump. We will also sometimes use the term $i$-clump to refer to the subtree of $T_{e}^{n}$ spanned by the points in an individual $i$-clump.

Contained within an $i$-clump there are several $j$-clumps where $j<i$. A $j$ spanning tree contained in an i-clump is a subtree spanned by choosing a point in each $j$-clump contained with $i$-clump. We will estimate the volume of $T_{e}^{n}$ by estimating the size and number of $i$-spanning trees contained in $T_{e}^{n}$.

Lemma 5.16. Let $\sigma: \Gamma \rightarrow \Gamma$ be as in Convention 5.4 and $f: T \rightarrow T$ a lift of $\sigma$. With the notation above, for any $j<i \leq n$, the number of $j$-clumps contained in an i-clump of $T_{e}^{n}$ is $\sim \lambda^{i-j}$.

Proof. As $\sigma: \Gamma \rightarrow \Gamma$ is irreducible and linearly expands any edge by the factor $\lambda$, there exist constants $K_{1} \geq 1$ and $C_{1} \geq 0$ such that for any point $x \in \Gamma$, we have $\frac{1}{K_{1}} \lambda^{\ell}-C_{1} \leq$ the number of points in $\sigma^{-\ell}(x) \leq K_{1} \lambda^{\ell}+C_{1}$.

Now after applying $f^{j}$ to $T$, any $j$-clump becomes a point and distinct $j$-clumps become distinct points. Further applying $f^{i-j}$, the image of the $j$-clumps which are contained within an $i$-clump all map to the same point $x$. Hence the $j$-clumps contained within an $i$-clump are parametrized by $\sigma^{-(i-j)}\left(p_{e}\right)$.

Lemma 5.17. Let $\sigma: \Gamma \rightarrow \Gamma$ be as in Convention 5.4 and $f: T \rightarrow T$ a lift of $\sigma$ and suppose that $\Gamma$ does not contain an indivisible orbit of periodic Nielsen paths. Fix 
an $\ell>0$, then for any $\ell<i \leq n$, the volume of $a(i-\ell)$-spanning tree contained in an $i$-clump is $\sim \mu^{i}$.

Proof. As in Lemma 5.16, the number of $(i-\ell)$-clumps contained in an $i$-clump is bounded above and below by some constants independent of $i$. Thus any $(i-\ell)-$ spanning tree is covered by a bounded number of $i$-vanishing paths. By Propositions 5.5 and 5.15 the length of an $i$-vanishing path is bounded above and below by linear functions of $\mu^{i}$.

Putting these together we can show:

Proposition 5.18. Let $\sigma: \Gamma \rightarrow \Gamma$ be as in Convention 5.4 and $f: T \rightarrow T$ a lift of $\sigma$ and suppose that $\Gamma$ does not contain an indivisible orbit of periodic Nielsen paths. Then for any edge $e \subset T$ :

$$
\operatorname{vol}\left(T_{e}^{n}\right) \sim \lambda^{n}+\lambda^{n-1} \mu+\cdots+\lambda \mu^{n-1}+\mu^{n} .
$$

Proof. As $T_{e}^{n}$ is covered by $i$-vanishing paths for $i \leq n$, we can also cover $T_{e}^{n}$ using $(i-1)$-spanning trees contained in $i$-clumps. The number of such $(i-1)$-spanning trees is equal to the number of $i$-clumps. Thus putting together Lemmas 5.16 and 5.17 we get that $\operatorname{vol}\left(T_{e}^{n}\right)$ is bounded above by a linear function of $\lambda^{n}+\lambda^{n-1} \mu+$ $\cdots+\lambda \mu^{n-1}+\mu^{n}$.

To get the lower bound we need to look carefully at the overlap of the these spanning trees. Let $K$ by the constant appearing in Proposition 5.5 and choose $\ell$ such that $\mu^{\ell}$ is much bigger than $K$. We will proceed by induction on $i$ and show that the volume of an $i$-clump is bounded below by a linear function of $\mu^{i}+\lambda^{\ell} \mu^{i-\ell}+\lambda^{2 \ell} \mu^{i-2 \ell}+\cdots$.

Fix an $i$-clump and let $T_{0}$ be an $(i-\ell)$-spanning tree contained within this $i$-clump. Let $j=i-\ell$ and denote the $j$-clumps contained within this $i$-clump by $T_{1}, \ldots, T_{M_{j}}$. By induction, the volume of any of the $T_{m}$ is bounded below by a linear function of $\mu^{j}+\lambda^{\ell} \mu^{j-\ell}+\lambda^{2 \ell} \mu^{j-2 \ell}+\cdots$. Notice that $M_{j} \approx \lambda^{\ell}$. For any $T_{m}$, the overlap of $T_{0}$ and $T_{m}$ has volume bounded above by $K \mu^{j}$. This follows as the overlap is contained within a $j$-vanishing path. Therefore the volume of $\widehat{T}_{0}$, the subforest of $T_{0}$ obtained by removing any overlap with some $T_{m}$ has volume bounded below by a linear function of $\mu^{i}$. Applying induction this shows that the volume of an $i$-clump is bounded below by a linear function of $\mu^{i}+\lambda^{\ell} \mu^{i-\ell}+\lambda^{2 \ell} \mu^{i-2 \ell}+\cdots \sim$ $\mu^{i}+\lambda \mu^{i-1}+\cdots+\lambda^{i-1} \mu+\lambda^{i}$. For $i=n$, we achieve the desired lower bound for $\operatorname{vol}\left(T_{e}^{n}\right)$.

Putting together Propositions 4.3 and 5.18 we get the second case of Theorem 1 from the Introduction.

Theorem 5.19. Suppose $\phi \in \operatorname{Out}\left(F_{k}\right)$ is a fully irreducible automorphism with expansion factor $\lambda$ and a nongeometric stable tree. Let $\mu$ be the expansion factor of $\phi^{-1}$. Then for any $T, T^{\prime} \in c v_{k}$ :

$$
i\left(T, T^{\prime} \phi^{n}\right) \sim \lambda^{n}+\lambda^{n-1} \mu+\cdots+\lambda \mu^{n-1}+\mu^{n} .
$$

5.2. $T^{+}$geometric. We will now look at the case when $T^{+}$is geometric. We need the following well-known result providing a special representative of an automorphism with geometric stable tree.

Proposition 5.20. Suppose that $T^{+}$, the stable tree of a fully irreducible automorphism $\phi$, is geometric. Then some power of $\phi$ has a train-track representative $\sigma: \Gamma \rightarrow \Gamma$ such that: 
1. $\sigma$ has exactly one indivisible Nielsen path;

2. $\sigma$ has a unique illegal turn;

3. $\sigma$ is homotopic to the result of iteratively folding the indivisible Nielsen path along the illegal turn.

Proof. Let $\sigma: \Gamma \rightarrow \Gamma$ be a stable train-track representative for $\phi$. Then $\sigma$ contains a unique indivisible orbit of periodic Nielsen paths, each path of which contains a unique illegal turn. This follows by Lemmas 3.4 and 3.9 of [4. Thus replacing $\sigma$ by a power, we can achieve 1. Further, by Lemma 3.9 of [4, after replacing $\sigma$ by this power, $\sigma: \Gamma \rightarrow \Gamma$ has a unique illegal turn, hence 2 holds.

Any homotopy equivalence between graphs factors into a sequence of folds 30 . Since edges in a legal turn do not get identified by $\sigma$, the first fold in the factored sequence is folding the indivisible Nielsen path. Folding a pair of edges of a stable train-track map results in a stable train-track map (remark on page 23 in [4). Hence the factored sequence is iteratively folding the indivisible Nielsen path.

We train-track representative satisfying the conclusions of the preceding proposition is called a parageometric train-track representative. For $t \geq 0$ let $\Gamma_{t}$ be the graph resulting from $\Gamma$ by iteratively folding the indivisible Nielsen path along the illegal turn at a constant rate, where for $t \in \mathbb{Z}_{\geq 0}$ the induced map $\sigma_{0 t}: \Gamma \rightarrow \Gamma_{t}$ is $\sigma^{t}$. For $0 \leq s \leq t$ let $\sigma_{s t}: \Gamma_{s} \rightarrow \Gamma_{t}$ by the induced map.

The dichotomy in Theorem 1 between geometric and nongeometric growth rates stems from the following observation. Suppose $\sigma: \Gamma \rightarrow \Gamma$ has an indivisible Nielsen path $\gamma$. For small $\epsilon>0$ let $\gamma_{\epsilon}$ be the subpath of $\gamma$ obtained by removing $\epsilon$ neighborhoods of its endpoints. Then $\gamma_{\epsilon}$ is an $i$-vanishing path for $\sigma$ and moreover, $i \rightarrow \infty$ as $\epsilon \rightarrow 0$. This observation follows as we can write $\gamma$ as a concatenation of legal paths $\gamma=a_{0} \cdot b_{0} \cdot \overline{b_{1}} \cdot \overline{b_{1}}$ where $\sigma\left(a_{i}\right)=a_{i} \cdot b_{i}$ and $\sigma\left(b_{0}\right)=\sigma\left(b_{1}\right)$. Hence for large $n$ (depending on $\epsilon$ ), $\left[\sigma^{n}\left(\gamma_{\epsilon}\right)\right] \subset b_{0} \cdot \overline{b_{1}}$ and therefore is an $n+1$-vanishing path. Therefore, in contrast with Lemma 5.9 and Proposition 5.15 in the nongeometric case, there are bounded length $i$-vanishing paths for large $i$. In fact, in the geometric case we claim that any $i$-vanishing path is a composition tightened relative to its endpoints of vanishing paths that are contained in this Nielsen path. A vanishing path contained in an indivisible Nielsen path is called a special vanishing path.

We begin with a simple criterion for finding special vanishing paths.

Lemma 5.21. Suppose $\sigma: \Gamma \rightarrow \Gamma$ is a parageometric train-track representative. There is a constant $T>0$ such that any vanishing path for $\sigma_{0 t}: \Gamma \rightarrow \Gamma_{t}$ for $t \leq T$ is a special vanishing path.

Proof. Choose $T$ such that $\sigma_{0 t}: \Gamma \rightarrow \Gamma_{t}$ does not identify a pair of vertices of $\Gamma$ for $t \leq T$. Then any vanishing path of $\sigma_{0 t}$ is contained within a pair of edges that are partially folded together by $\sigma_{0 t}$, and the turn between these edges is illegal. As $\sigma$ has a unique illegal turn and this illegal turn is contained in the unique Nielsen path, any vanishing path for $\sigma_{0 t}$ is contained in the Nielsen path and hence is a special vanishing path.

We can now show that vanishing paths can be covered by special vanishing paths.

Proposition 5.22. Suppose $\sigma: \Gamma \rightarrow \Gamma$ is a parageometric train-track representative and $\gamma$ is $i$-vanishing path for $\sigma$. Then $\gamma=\left[\gamma_{1} \cdots \gamma_{\ell}\right]$ where for $j=1, \ldots, \ell$, the path $\gamma_{j}$ is a special vanishing path. 
Proof. Since folding $\Gamma$ does not collapse loops and since $\Gamma$ has a unique illegal turn, for any path $\alpha$ in $\Gamma$, there is a lower bound on the distance between two illegal turns in $\sigma_{0 t}(\alpha)$ independent of $\alpha$ and $t$. Therefore, there is an $\epsilon$ such that if $\alpha$ is a vanishing path with length $(\alpha)<\epsilon$ then $\alpha$ contains a single illegal turn. Hence, there is a $\delta>0$ such that if $\gamma$ is a vanishing path for $\sigma_{t}: \Gamma \rightarrow \Gamma$ with $t$ minimal, then $\left[\sigma_{0 t-\delta}(\gamma)\right]$ contains a single illegal turn.

We will prove the proposition by induction. The inductive claim is as follows: if $\gamma$ is a vanishing path for $\sigma_{0 t_{\gamma}}: \Gamma \rightarrow \Gamma_{t_{\gamma}}$ with $t_{\gamma}$ minimal, then $\gamma=\left[a_{1} \cdot a_{0} \cdot a_{2}\right]$ where $a_{0}$ is a special vanishing path and $a_{1}$ and $a_{2}$ are vanishing paths for $\sigma_{0 t_{1}}: \Gamma \rightarrow \Gamma_{t_{1}}$ where $t_{1}=t_{\gamma}-\delta$. The basecase, where $t_{\gamma} \leq T$ where $T$ is the constant from Lemma 5.21 is proved by Lemma 5.21 as in this case $\gamma=a_{0}$ is a special vanishing path.

By construction a single illegal turn in $\gamma$ is still present in $\left[\sigma_{0, t_{1}}(\gamma)\right]$. In the universal cover of $\Gamma$ there are lifts of the vanishing path $\gamma$ and the Nielsen path that share a lift of this illegal turn. Write the lift of $\gamma$ as $\gamma_{1} \cdot \gamma_{0} \cdot \gamma_{2}$ where $\gamma_{0}$ is the common overlap between the lift of $\gamma$ and the lift of the Nielsen path. This decomposes the lift of the Nielsen path into $\beta_{1} \cdot \gamma_{0} \cdot \beta_{2}$. Notice that $\beta_{1}$ and $\beta_{2}$ are legal paths.

Since $\left[\sigma_{0 t_{1}}(\gamma)\right]$ only contains a single illegal turn, as in Lemma 5.21 we see that $\left[\sigma_{0 t_{1}}(\gamma)\right]$ is contained in the image of the Nielsen path in $\Gamma_{t_{1}}$. Therefore, we can find a subpath $\beta_{1}^{\prime}$ of $\beta_{1}$ such that $\gamma_{1} \cdot \overline{\beta_{1}^{\prime}}$ is a vanishing path for $\sigma_{0 t_{1}}$. Similarly we can find a subpath $\beta_{2}^{\prime}$ of $\beta_{2}$ such that $\gamma_{2} \cdot \overline{\beta_{2}^{\prime}}$ is also a vanishing path for $\sigma_{0 t_{1}}$. Then $\beta_{1}^{\prime} \cdot \gamma_{0} \cdot \beta_{2}^{\prime}$ is a special vanishing path. See Figure 4. By induction, we know that

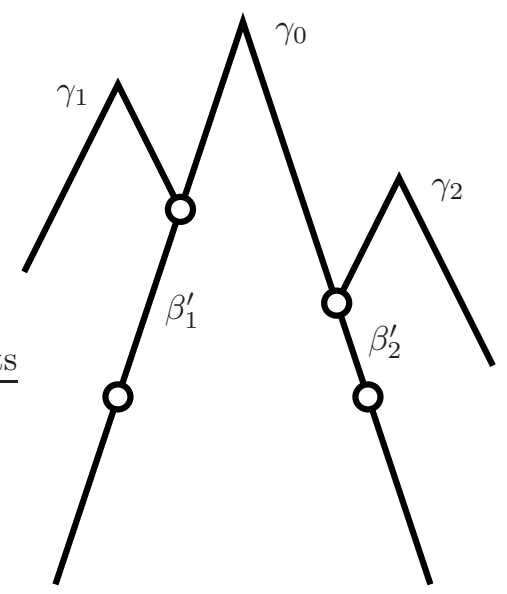

Figure 4. Decomposing the vanishing path $\gamma$ in the proof of Proposition 5.22

both $\gamma_{1} \cdot \overline{\beta_{1}^{\prime}}$ and $\gamma_{2} \cdot \overline{\beta_{2}^{\prime}}$ are the compositions of special vanishing paths pulled tight. Therefore, $\gamma$ is the composition of special vanishing paths pulled tight.

We can now prove the first case of Theorem 1 from the Introduction.

Theorem 5.23. Suppose $\phi \in \operatorname{Out}\left(F_{k}\right)$ is a fully irreducible automorphism with expansion factor $\lambda$ and a geometric stable tree. Then for any $T^{\prime}, T^{\prime \prime} \in c v_{k}$ :

$$
i\left(T^{\prime}, T^{\prime \prime} \phi^{n}\right) \sim \lambda^{n} .
$$


Proof. First suppose that $\phi$ is represented by a parageometric train-track map $\sigma: \Gamma \rightarrow \Gamma$. This lifts to a map $f: T \rightarrow T$. By Proposition $4.3 i\left(T^{\prime}, T^{\prime \prime} \phi^{n}\right) \sim \operatorname{vol}\left(T_{e}^{n}\right)$ where $T_{e}^{n} \subset T$ is the subtree spanned by the points in $\left(f^{n}\right)^{-1}\left(p_{e}\right)$ for any edge $e \subset T$. Then $T_{e}^{n}$ as is a union of $i$-vanishing paths for $i \leq n$ and by Proposition 5.22 it is also covered special vanishing paths. As in Lemma 5.16 the number of special vanishing paths needed to cover $T_{e}^{n}$ is $\sim \lambda^{n}$.

If $\phi$ is not represented by a parageometric train-track map, then by Proposition 5.20 some power $\phi^{\ell}$ is. Then for any $i$ we have $i\left(T^{\prime}, T^{\prime \prime} \phi^{i+n \ell}\right)=i\left(T^{\prime},\left(T^{\prime \prime} \phi^{i}\right) \phi^{n \ell}\right) \sim$ $\left(\lambda^{\ell}\right)^{n} \sim \lambda^{i+n \ell}$, hence $i\left(T^{\prime}, T^{\prime \prime} \phi^{n}\right) \sim \lambda^{n}$.

Combining Theorems 5.19 and 5.23 we get Theorem 1 from the Introduction. We conclude with an example of a parageometric automorphism, illustrating the difference between the length of vanishing paths in the geometric direction and nongeometric direction.

Example 5.24. In this example we present subtrees $T_{a}^{n}$ for the following fully irreducible automorphisms:

$$
\begin{array}{rlrl}
a & \mapsto a c & a & \mapsto b \\
\phi: b & \mapsto a & \forall: b & \mapsto b c \\
c & \mapsto b & c & \mapsto a b
\end{array}
$$

As in Example 3.10 we let $T$ be the universal cover of $R_{3}$, the 3-rose marked with petals labeled $a, b, c$ and let $f_{\phi}: T \rightarrow T$ and $f_{\psi}: T \rightarrow T$ denote the lift of the obvious homotopy equivalences of $R_{3}$ representing $\phi$ and $\psi$ respectively. Figure 5 shows $\left(f_{\phi}^{n}\right)^{-1}\left(p_{a}\right)$ and Figure 6 shows $\left(f_{\psi}^{n}\right)^{-1}\left(p_{a}\right)$ for $n=6,7,8,9,10$. Notice how the points in $\left(f^{n}\right)^{-1}\left(p_{a}\right)$ stay uniformly close together (since the stable tree for $\phi$ is geometric) where as in $\left(f_{\psi}^{n}\right)^{-1}\left(p_{a}\right)$ they start to clump together (since the stable tree for $\psi$ is nongeometric).

\section{REFERENCES}

[1] J. Behrstock, B. Kleiner, Y. Minsky, And L. Mosher, Geometry and rigidity of mapping class groups. arXiv:0801.2006

[2] M. Bestvina And M. Feighn, Outer limts. preprint (1992).

[3] M. Bestvina, M. Feighn, And M. Handel, Laminations, trees, and irreducible automorphisms of free groups, Geom. Funct. Anal., 7 (1997), pp. 215-244.

[4] M. Bestvina And M. Handel, Train tracks and automorphisms of free groups, Ann. of Math. (2), 135 (1992), pp. 1-51.

[5] F. Bonahon, Bouts des variétés hyperboliques de dimension 3, Ann. of Math. (2), 124 (1986), pp. $71-158$.

[6] B. H. Bowditch, Intersection numbers and the hyperbolicity of the curve complex, J. Reine Angew. Math., 598 (2006), pp. 105-129.

[7] M. R. BRidson And K. Vogtmann, Automorphism groups of free groups, surface groups and free abelian groups, in Problems on mapping class groups and related topics, vol. 74 of Proc. Sympos. Pure Math., Amer. Math. Soc., Providence, RI, 2006, pp. 301-316.

[8] D. Cooper, Automorphisms of free groups have finitely generated fixed point sets, J. Algebra, 111 (1987), pp. 453-456.

[9] M. Culler, G. Levitt, and P. Shalen. unpublished manuscript.

[10] M. Culler and J. W. Morgan, Group actions on R-trees, Proc. London Math. Soc. (3), 55 (1987), pp. 571-604.

[11] M. Culler and K. Vogtmann, Moduli of graphs and automorphisms of free groups, Invent. Math., 84 (1986), pp. 91-119.

[12] K. Fujiwara and P. Papasoglu, JSJ-decompositions of finitely presented groups and complexes of groups, Geom. Funct. Anal., 16 (2006), pp. 70-125. 
GROWTH OF INTERSECTION NUMBERS FOR FREE GROUP AUTOMORPHISMS

31

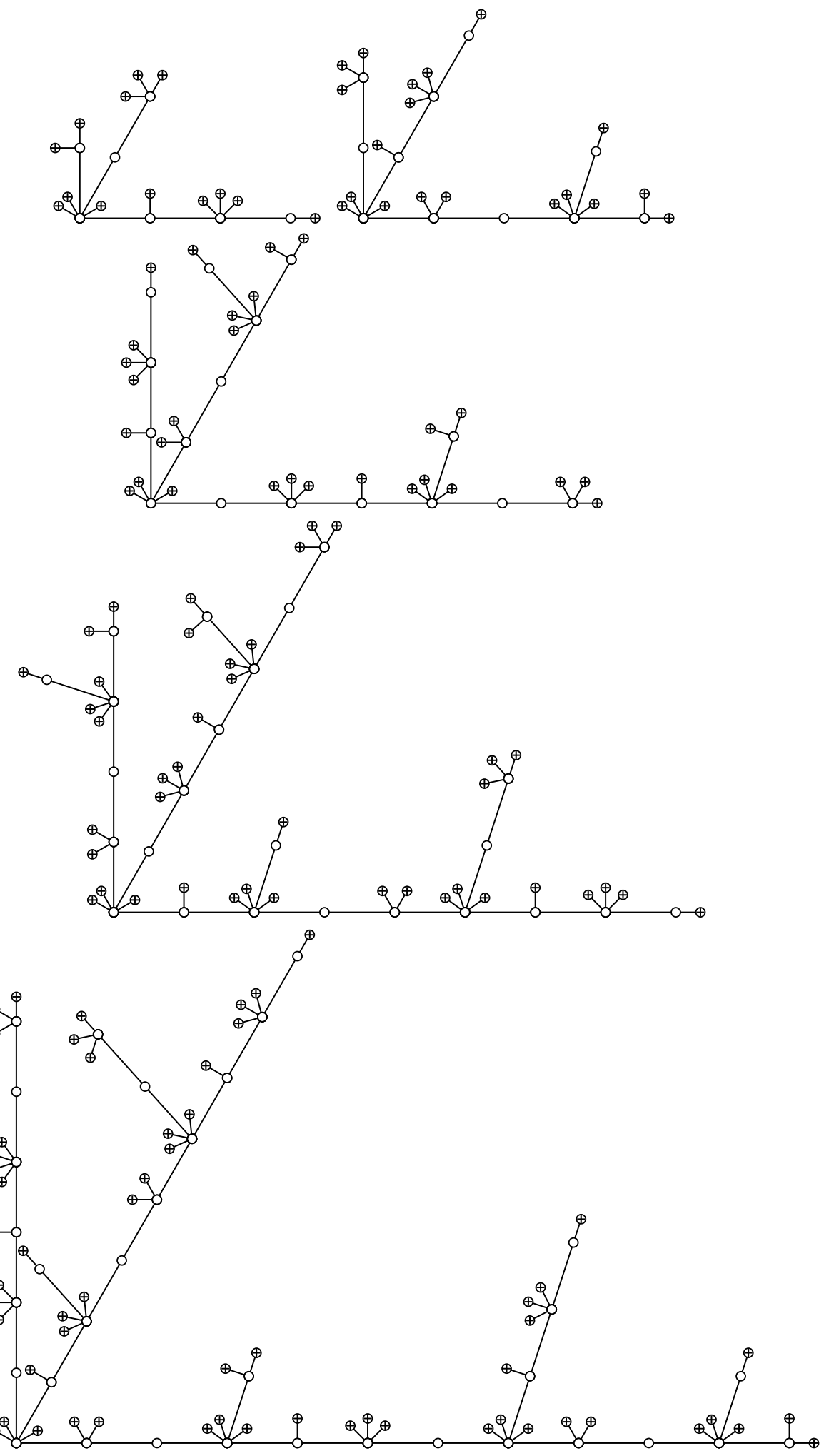

Figure 5. The trees $T_{a}^{n}$ in Example 5.24 for the automorphism with geometric stable tree. 


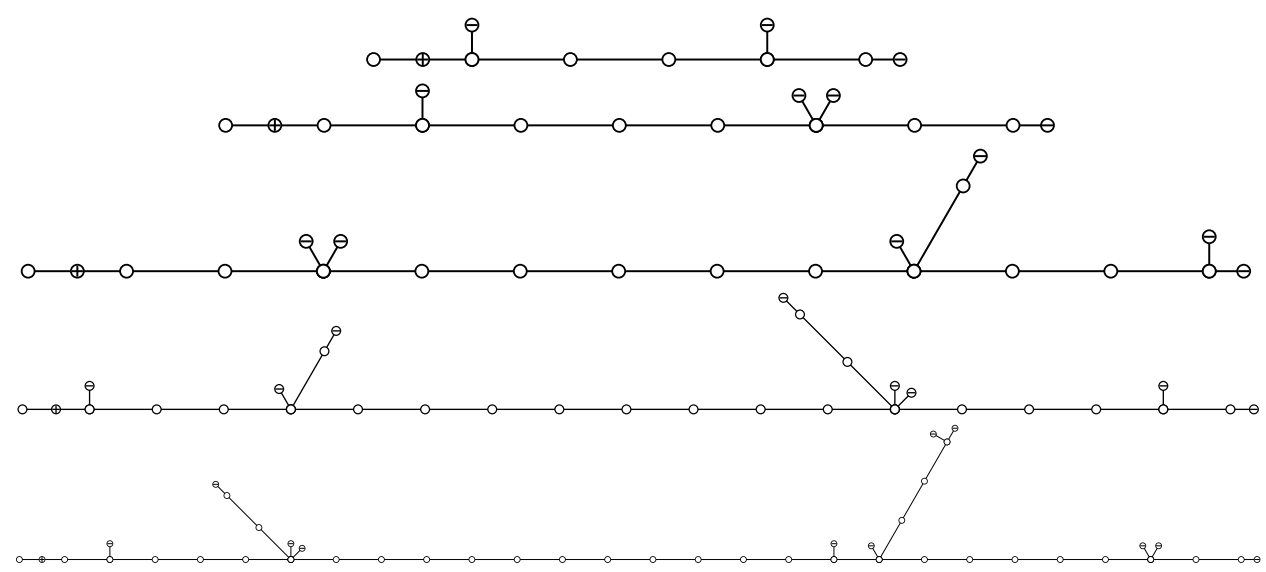

Figure 6. The trees $T_{a}^{n}$ in Example 5.24 for the automorphism with nongeometric stable tree.

[13] V. Guirardel, Cour et nombre d'intersection pour les actions de groupes sur les arbres, Ann. Sci. École Norm. Sup. (4), 38 (2005), pp. 847-888.

[14] M. Handel And L. Mosher, The expansion factors of an outer automorphism and its inverse, Trans. Amer. Math. Soc., 359 (2007), pp. 3185-3208 (electronic).

[15] — Parageometric outer automorphisms of free groups, Trans. Amer. Math. Soc., 359 (2007), pp. 3153-3183 (electronic).

[16] J. L. HARER, Stability of the homology of the mapping class groups of orientable surfaces, Ann. of Math. (2), 121 (1985), pp. 215-249.

[17] - Stability of the homology of the mapping class groups of orientable surfaces, Ann. of Math. (2), 121 (1985), pp. 215-249.

[18] - The virtual cohomological dimension of the mapping class group of an orientable surface, Invent. Math., 84 (1986), pp. 157-176.

[19] W. J. HARvey, Boundary structure of the modular group, in Riemann Surfaces and Related Topics: Proceedings of the 1978 Stony Brook Conference, I. Kra and B. Maskit, eds., Ann. of Math. Stud. 97, Princeton, 1981.

[20] A. Hatcher and K. Vogtmann, The complex of free factors of a free group, Quart. J. Math. Oxford Ser. (2), 49 (1998), pp. 459-468.

[21] N. V. IVAnov, Automorphisms of complexes of curves and of Teichmüller spaces, Internat. Math. Res. Notices, (1997), pp. 651-666.

[22] I. KAPOVICH, Currents on free groups, in Topological and asymptotic aspects of group theory, vol. 394 of Contemp. Math., Amer. Math. Soc., Providence, RI, 2006, pp. 149-176.

[23] I. Kapovich AND M. Lustig, Geometric intersection number and analogues of the curve complex for free groups, Geom. Topol., 13 (2009), pp. 1805-1833 (electronic).

[24] M. Korkmaz, Automorphisms of complexes of curves on punctured spheres and on punctured tori, Topology Appl., 95 (1999), pp. 85-111.

[25] G. LevitT And F. Paulin, Geometric group actions on trees, Amer. J. Math., 119 (1997), pp. $83-102$.

[26] F. Luo, Automorphisms of the complex of curves, Topology, 39 (2000), pp. 283-298.

[27] H. A. Masur and Y. Minsky, Geometry of the complex of curves I: Hyperbolicity, Invent. Math., 138 (1999), pp. 103-149.

[28] P. Sсотт, The symmetry of intersection numbers in group theory, Geom. Topol., 2 (1998), pp. 11-29 (electronic).

[29] P. Scott And G. A. Swarup, Splittings of groups and intersection numbers, Geom. Topol., 4 (2000), pp. 179-218 (electronic).

[30] J. R. Stallings, Topology of finite graphs, Invent. Math., 71 (1983), pp. 551-565.

[31] W. P. Thurston, On the geometry and dynamics of diffeomorphisms of surfaces, Bull. Amer. Math. Soc. (N.S.), 19 (1988), pp. 417-431. 
Dept. of Mathematics, Lehman College, Bronx, NY 10468

E-mail address: jason.behrstock@lehman.cuny.edu

Dept. of Mathematics, University of Utah, 155 South 1400 East, JWB 233, Salt Lake City, UT 84102

E-mail address: bestvina@math.utah.edu

Dept. of Mathematics, University of Oklahoma, Norman, OK 73019

E-mail address: mclay@math.ou.edu 


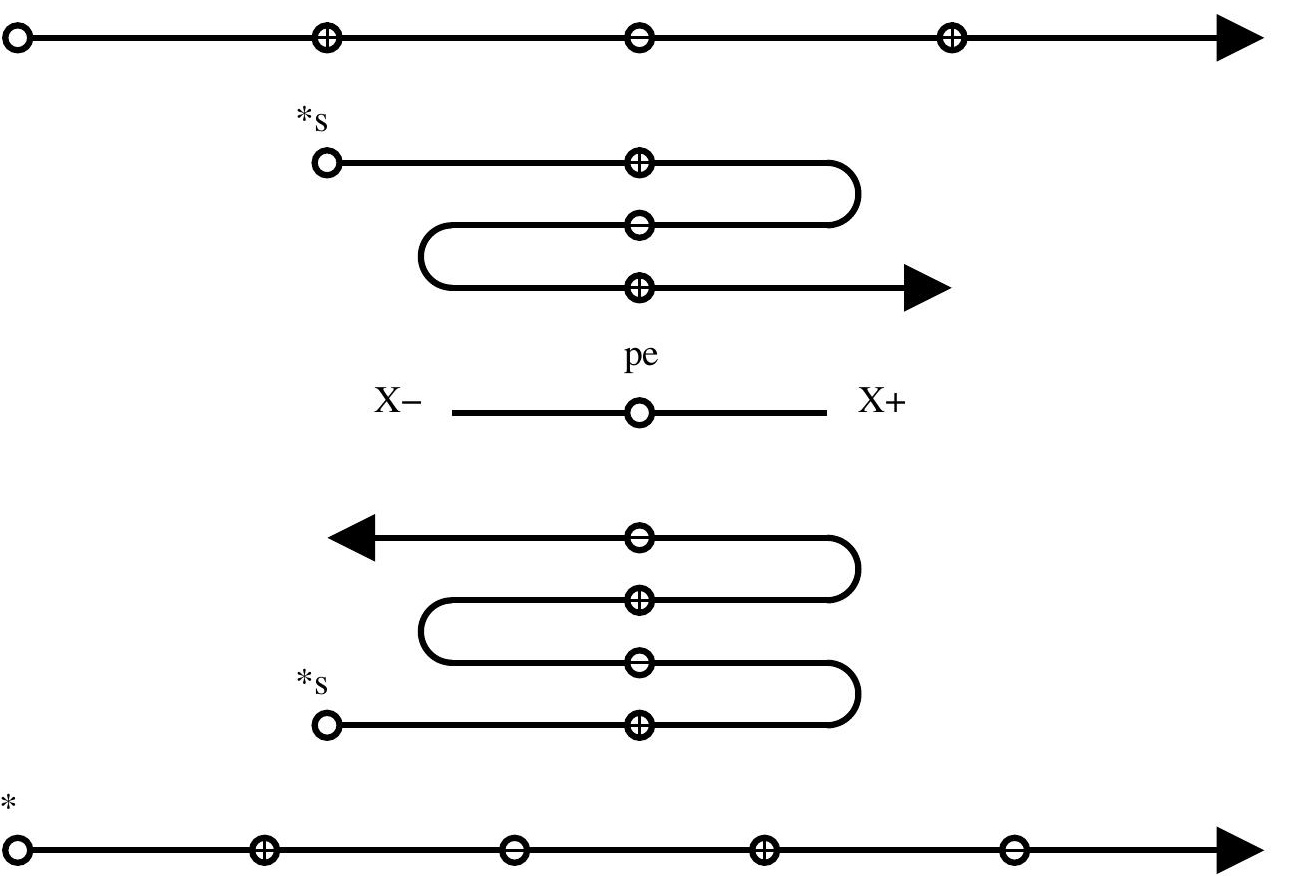

